ESAIM: COCV 27 (2021) 25

https://doi.org/10.1051/cocv/2021018
ESAIM: Control, Optimisation and Calculus of Variations

www.esaim-cocv.org

\title{
ON THE CONVEXITY CONDITION FOR THE SEMI-GEOSTROPHIC SYSTEM
}

\author{
ADRIAN TUDORASCU*
}

\begin{abstract}
We show that conservative distributional solutions to the Semi-Geostrophic systems in a rigid domain are in some well-defined sense critical points of a time-shifted energy functional involving measure-preserving rearrangements of the absolute density and momentum, which arise as one-parameter flow maps of continuously differentiable, compactly supported divergence free vector fields. We also show directly, with no recourse to Monge-Kantorovich theory, that the convexity requirement on the modified pressure potentials arises naturally if these critical points are local minimizers of said energy functional for any admissible vector field. The obligatory connection with the Monge-Kantorovich theory is addressed briefly.
\end{abstract}

Mathematics Subject Classification. 35A15, 35E10, 35F20, 35Q35.

Received June 18, 2020. Accepted February 8, 2021.

\section{INTRODUCTION}

The Semi-Geostrophic (abbreviated SG in this paper) system is a model of large scale atmosphere/ocean flows, where "large-scale" means that the flow is rotation-dominated [18]:

$$
\begin{array}{ll}
D_{t} \mathbf{u}_{g}+\bar{\nabla} p+J \mathbf{u}=0 & \\
\mathbf{u}_{g}=J \nabla p, D_{t} \partial_{3} p=0 & \text { in }[0, T) \times \Omega, \\
\nabla \cdot \mathbf{u}=0, & \text { on }(0, T) \times \partial \Omega \\
\mathbf{u} \cdot \nu=0 & \text { in } \Omega, \\
p(0, \cdot)=p_{0}
\end{array}
$$

where $\Omega \subset \mathbb{R}^{3}$ is open and bounded, $0<T<\infty, \nabla$ denotes the spatial gradient, $\bar{\nabla}=\left(\partial_{1}, \partial_{2}, 0\right), \nabla \cdot$ the spatial divergence, $D_{t}:=\partial_{t}+\mathbf{u} \cdot \nabla$ and $\nu$ is the (outward) unit vector normal to $\Omega$. The total wind velocity is $\mathbf{u}$, while $\mathbf{u}_{g}$ is the geostrophic wind. Here,

$$
J=\left(\begin{array}{rrr}
0 & -1 & 0 \\
1 & 0 & 0 \\
0 & 0 & 0
\end{array}\right)
$$

Keywords and phrases: Semi-Geostrophic System, Cullen-Purser stability, modified pressure, convexity of the potential.

Department of Mathematics, West Virginia University, Morgantown, WV 26506, USA.

* Corresponding author: adriant@math.wvu.edu 
There are many works dedicated to solutions $(p, \mathbf{u})$ that satisfy the Cullen-Purser stability condition (see, e.g., $[9,12])$. This amounts to imposing that $P(t, x):=p(t, x)+\left(x_{1}^{2}+x_{2}^{2}\right) / 2$ be convex as a function of $x=$ $\left(x_{1}, x_{2}, x_{3}\right) \in \Omega$ for all $t \in[0, T)$. Our main goal in this paper is to provide a rigorous justification for imposing convexity on the modified pressure map $P$.

In terms of $(P, \mathbf{u}),(1.1)$ can be written

$$
\begin{array}{ll}
D_{t} X=J(X-x) & \\
X=\nabla P & \text { in }[0, T) \times \Omega, \\
\nabla \cdot \mathbf{u}=0 & \text { on }(0, T) \times \partial \Omega, \\
\mathbf{u} \cdot \nu=0 & \text { in } \Omega,
\end{array}
$$

If $\left.\nabla P(t, \cdot)_{\#} \mathcal{L}^{3}\right|_{\Omega}=: \rho(t, \cdot)$, then one can use the Legendre-Fenchel transform

$$
P^{*}(t, \cdot):=\sup _{x \in \Omega}\{x \cdot X-P(t, x)\}
$$

of $P(t, \cdot)$ to formally rewrite $(1.1)$ as the so-called SG in dual variables

$$
\begin{aligned}
& \partial_{t} \rho+\nabla \cdot(U \rho)=0 \quad \text { in }[0, T) \times \Omega, \\
& \left.\nabla P(t, \cdot)_{\#} \mathcal{L}^{3}\right|_{\Omega}=\rho(t, \cdot) \quad \text { for any } t \in[0, T) \\
& U(t, X)=J\left[X-\nabla P^{*}(t, X)\right] \\
& \rho(0, X)=\rho_{0}(X) \quad \text { for a.e. } X \in \Omega .
\end{aligned}
$$

In [15] one can find a comprehensive bibliography on solutions of SG in dual space; a first result on existence of solutions in dual variables was obtained in [3], where the system was treated as a Monge-Ampere equation coupled with a transport equation. Generally, these solutions are not known to be regular enough to be translated into Eulerian solutions of the problem in physical space. Cullen and Feldman [11] pulled the dual space solutions back to physical space in the form of weak Lagrangian solutions in the case $\rho_{0} \in L^{p}\left(\mathbb{R}^{3}\right)$ for some $p>1$; the question left open in its generalization (to $p \geq 1$ ) by Faria et al. [14] regards the even more general case, i.e. the case in which $\rho_{0}$ may be a singular probability measure. We settled that in [16], where we introduced an appropriate generalization of weak Lagrangian solutions, namely renormalized relaxed Lagrangian solutions. More recently, existence of Eulerian solutions for a class of initial data, where the conditions include the requirement that the support of $\rho_{0}=\left.\nabla P_{0 \#} \mathcal{L}^{3}\right|_{\Omega}$ in the dual space is the whole space, was obtained by Ambrosio et al. $[1,2]$ based on the results of De Philippis and Figalli [13] on regularity of solutions for the Monge-Ampere equation.

Loeper [20] first proved uniqueness for classical solutions in the class of functions which are Hölder continuous in space uniformly with respect to time. Cheng, Cullen and Feldman [7], besides the short time existence of classical solutions for SG with variable Coriolis force, prove uniqueness of such solutions in the class of sufficiently regular classical solutions. There is also work by Brenier and Cullen [5] where a formal proof of convergence of solutions for a Navier-Stokes with Boussinesq approximation to a solution of $x-z \mathrm{SG}$ is given. Both systems are assumed to have smooth solutions, which is unknown in general even for "nice" initial data. Also, it is assumed that $P(t, \cdot)$ has a smooth convex extension to $\mathbb{R}^{3}$ such that $\nabla^{2} P^{*}(t, \cdot)$ is bounded away from zero and infinity uniformly for $t \in[0, T]$ (for some $T>0$ ). A weak-strong uniqueness result in the case of uniformly convex $P^{*}$ was recently obtained in [17] based on the relative entropy approach from [5]. This makes an even stronger case for the convexity principle, as it is shown in [17] that solutions satisfying it are stable in the usual sense (small perturbations in the initial data lead to small perturbations at later times).

In this paper we introduce a rigorous derivation of the Cullen-Purser stability condition by first observing that classical solutions are "dynamical" critical points in some suitable sense of the SG energy functional. More 
precisely, let $t \geq 0$ and

$$
E(t):=\int_{\Omega}|X(t, x)-x|^{2} \mathrm{~d} x
$$

where $X$ solves (1.2). It is easy to show that classical solutions to (1.2) conserve (1.4), i.e. $E(t)=E(0)$ for all $t>0$. Moreover, in [16] it is shown that some "very weak" solution corresponding to any $P_{0} \in H^{1}(\Omega)$ does exist, satisfy $P(t, \cdot) \in H^{1}(\Omega)$ and conserve the energy for all $t \geq 0$. Therefore, Theorem 2.2 below applies to such solutions.

In [10] the authors use a definition of stability for the free-surface SG system based on an extant notion (due to Giaquinta and Hildebrand) of inner-variation for the energy functional. In the end, the convexity of $P(t, \cdot)$ follows readily from the nonnegativity of the second variation, which in their case amounts to

$$
\int_{\Omega} \nabla^{2} P(t, x) \Psi(x) \cdot \Psi(x) \mathrm{d} x \geq 0 \text { for all } t>0 \text { and all } \Psi \in C_{c}^{\infty}\left(\Omega ; \mathbb{R}^{3}\right) .
$$

For any $\mathbf{u} \in \mathbb{R}^{3}, x_{0} \in \Omega$ and $r>0$ sufficiently small one approximates $\mathbf{u}$ times the indicator function of $B\left(x_{0}, r\right)$ in $L^{2}\left(\Omega ; \mathbb{R}^{3}\right)$ by $C_{c}^{\infty}\left(\Omega ; \mathbb{R}^{3}\right)$ vector functions to conclude that

$$
\mathbf{u}^{T}\left[\int_{B\left(x_{0}, r\right)} \nabla^{2} P(t, x) \mathrm{d} x\right] \mathbf{u} \geq 0
$$

which, after dividing by the volume of $B\left(x_{0}, r\right)$ and letting $r \rightarrow 0^{+}$, yields the nonnegative definiteness of $\nabla^{2} P\left(t, x_{0}\right)$ (for a.e. $x_{0} \in \Omega$ if $\nabla^{2} P(t, \cdot)$ is only assumed in $L^{1}$ ). So, imposing vanishing first inner-variation and nonnegative second variation does single out solutions satisfying the convexity property, but this selection criterion has one drawback: note that the inner-variation of the energy functional involves the composition of $X(t, \cdot)$ with diffeomorphisms that are not necessarily preserving the Lebesgue measure restricted to $\Omega$; this composition does not preserve the dual-space measures associated to the physical space problem, i.e. it is not a measure-preserving rearrangement of the absolute momentum $\left(u_{g, 1}-x_{2}, u_{g, 2}+x_{1}\right)=\left(-\partial_{2} P, \partial_{1} P\right)$ and density $\rho=\partial_{3} p=\partial_{3} P$. Indeed, the variation of $X(t, \cdot)=\nabla P(t, \cdot)$ is taken [10] of the form $X\left(t, \Phi^{\varepsilon}(x)\right)$, where $\Phi^{\varepsilon}=\operatorname{Id}+\varepsilon \Phi$ is a smooth and compactly supported perturbation of the identity map, so it is measure preserving only in the limit as $\varepsilon \rightarrow 0^{+}$(when it becomes the identity map). Our approach remedies that in Section 2, in that (a) it first shows that all solutions to SG are "dynamical" critical points for the "time-shifted" energy functional rearranged via the composition with one-parameter families of measure-preserving maps which are flow maps of $C^{1}$ compactly supported divergence free vector fields $\mathbf{v}$, and (b) it then shows that these critical points correspond to minima of the shifted functional if and only if the maps $P(t, \cdot)$ are convex. The trade-off is a more elaborate proof of convexity, owing to the fact that the admissible $\mathbf{v}$ are subject to the restriction $\nabla \cdot \mathbf{v} \equiv 0$ in $\Omega$ even if they are chosen to have compact support (such as $\Psi$ above).

Section 3 discusses some obligatory connections with the Optimal Transport theory, while the Appendix contains self-contained proofs (that may be hard to find) of some fairly well-known results, along with different proofs of the convexity result in the $2 \mathrm{D}$ and $3 \mathrm{D}$ cases.

\section{MAIN RESUlts}

Assume $\Omega \in C^{1}$ and let

$$
\mathcal{V}(\mathbb{R} ; \Omega):=\left\{\mathbf{v} \in C^{1}\left(\mathbb{R} \times \bar{\Omega} ; \mathbb{R}^{3}\right): \nabla_{x} \cdot \mathbf{v} \equiv 0 \text { in } \mathbb{R} \times \Omega \text { and } \mathbf{v} \cdot \nu \equiv 0 \text { on } \mathbb{R} \times \partial \Omega\right\}
$$


and

$$
\mathcal{V}(\Omega):=\left\{\mathbf{v} \in C_{c}^{1}\left(\Omega ; \mathbb{R}^{3}\right): \nabla \cdot \mathbf{v} \equiv 0 \text { in } \Omega\right\}
$$

It is well-known (also, see Appendix) that for any $t \in \mathbb{R}$ any $\mathbf{v} \in \mathcal{V}(\mathbb{R} ; \Omega)$ introduces a one-parameter family of measure-preserving diffeomorphisms $\mathbb{R} \ni s \rightarrow g(s ; t, \cdot) \in \operatorname{Diff}(\bar{\Omega})$ given by

$$
\begin{aligned}
\partial_{s} g(s ; t, x) & =\mathbf{v}(s, g(s ; t, x)) & & \text { for all }(s, x) \in \mathbb{R} \times \bar{\Omega}, \\
g(t ; t, x) & =x & & \text { for all } x \in \bar{\Omega} .
\end{aligned}
$$

We will adopt the notation $g[\mathbf{v}]$ for the solution of the above problem in order to emphasize that it is completely determined by the choice of $\mathbf{v} \in \mathcal{V}(\mathbb{R} ; \Omega)$. With this in hand, let us define, for all $t \geq 0$ and all $\mathbf{v} \in \mathcal{V}(\mathbb{R} ; \Omega)$,

$$
J[t, \mathbf{v}](s):=\int_{\Omega}|X(s+t, g[\mathbf{v}](s+t ; t, x))-x|^{2} \mathrm{~d} x, \quad s \in \mathbb{R}, s \geq-t .
$$

The following theorem lies at the core of our approach.

Theorem 2.1. Let $(P, \mathbf{u})$ be a classical solution for (1.2). For any $t \geq 0$ and $\mathbf{v} \in \mathcal{V}(\mathbb{R} ; \Omega)$, the function $J[t, \mathbf{v}]$ has a critical point at $s=0$.

Proof. We differentiate $J[t, \mathbf{v}]$ with respect to $s$ and use (2.1) to get

$$
\begin{aligned}
& \frac{\mathrm{d}}{\mathrm{d} s} J[t, \mathbf{v}](s)=2 \int_{\Omega}\left[\partial_{t} X(s+t, g(s+t ; t, x))\right. \\
& \quad+\nabla X(s+t, g(s+t ; t, x)) \mathbf{v}(s+t, g(s+t ; t, x))] \cdot[X(s+t, g(s+t ; t, x))-x] \mathrm{d} x,
\end{aligned}
$$

where we simplified the notation $g[\mathbf{v}]$ to $g$ (as we will often do when there is no confusion about the relationship between $\mathbf{v}$ and $g$ ). In light of the initial condition in (2.1), we have

$$
\left.\frac{\mathrm{d}}{\mathrm{d} s} J[t, \mathbf{v}]\right|_{s=0}=2 \int_{\Omega}\left[\partial_{t} X(t, x)+\nabla X(t, x) \mathbf{v}(t, x)\right] \cdot[X(t, x)-x] \mathrm{d} x .
$$

We next employ (1.2) in order to replace

$$
\partial_{t} X(t, x)=-\nabla X(t, x) \mathbf{u}(t, x)+J[X(t, x)-x]
$$

so we are led to

$$
\left.\frac{\mathrm{d}}{\mathrm{d} s} J[t, \mathbf{v}]\right|_{s=0}=2 \int_{\Omega} \nabla X(t, x)[\mathbf{v}(t, x)-\mathbf{u}(t, x)] \cdot[X(t, x)-x] \mathrm{d} x .
$$

The identity

$$
\nabla[Y \cdot Z]=(\nabla Y)^{T} Z+(\nabla Z)^{T} Y
$$

yields

$$
\nabla\left[|X|^{2}\right]=2(\nabla X)^{T} X \text { and } \nabla[x \cdot X]=X+(\nabla X)^{T} x,
$$


so the fact that $\mathbf{v}(t, \cdot)-\mathbf{u}(t, \cdot)$ is divergence free with no flux through the boundary implies $J[t, \mathbf{v}]^{\prime}(0)=0$ provided that $X(t, \cdot)$ is a gradient, which it is.

It is then natural to ask if certain solutions of (1.2) render $s=0$ a (local) minimum point for $J[t, \mathbf{v}]$ for all $t \geq 0$ and all $\mathbf{v} \in \mathcal{V}(\mathbb{R} ; \Omega)$. As we shall soon see, this is equivalent to the convexity of $P(t, \cdot)$ (and so, according to the Optimal Transport theory, the assumed local minimum is, in fact, global).

Theorem 2.2. Let $(P, \mathbf{u})$ be a conservative (i.e., $E(t)=E(0)$ for all $t \geq 0$ ) distributional solution for (1.2) which satisfies $P(t, \cdot) \in W^{1,1}(\Omega)$ for all $t \geq 0$. Assume that for any $t>0$ and $\mathbf{v} \in \mathcal{V}(\Omega)$, the function $J[t, \mathbf{v}]$ has a minimum at $s=0$ over the interval $(-t, \infty)$. Then

$$
\int_{\Omega} P(t, x) \nabla \cdot[\nabla \mathbf{v}(x) \mathbf{v}(x)] \mathrm{d} x \geq 0 \text { for all } t \geq 0 \text { and all } \mathbf{v} \in \mathcal{V}(\Omega)
$$

Proof. Since $\mathbf{v}$ is time-independent, we have $g[\mathbf{v}](s+t ; t, x)=g(s, x)$, where

$$
\partial_{s} g(s, x)=\mathbf{v}(g(s, x)) \text { in } \mathbb{R} \times \Omega, g(0, x)=x \text { in } \Omega .
$$

Thus, we have

$$
J[t, \mathbf{v}](s)=\int_{\Omega}|X(s+t, g(s, x))-x|^{2} \mathrm{~d} x \geq \int_{\Omega}|X(t, x)-x|^{2} \mathrm{~d} x=E(t)
$$

for all $t>0$ and all $s>-t$. By the conservation of energy, $E(t)=E(s+t)$ for all $s \geq-t$, so

$$
\int_{\Omega}|X(s+t, g(s, x))-x|^{2} \mathrm{~d} x \geq \int_{\Omega}|X(s+t, x)-x|^{2} \mathrm{~d} x
$$

for all $t>0$ and $s>-t$. We let $s+t=: \tau>0$, so $s=\tau-t$ and

$$
\int_{\Omega}|X(\tau, g(\tau-t, x))-x|^{2} \mathrm{~d} x \geq \int_{\Omega}|X(\tau, x)-x|^{2} \mathrm{~d} x
$$

for all $t>0$ and $\tau>0$, or, equivalently,

$$
\int_{\Omega}|X(\tau, g(s, x))-x|^{2} \mathrm{~d} x \geq \int_{\Omega}|X(\tau, x)-x|^{2} \mathrm{~d} x
$$

for all $\tau>0$ and all $s<\tau$. We revert back to the notation $\tau=: t$, fix such $t>0$, so that

$$
i(s):=\int_{\Omega}|X(t, g(s, x))-x|^{2} \mathrm{~d} x
$$

achieves a local minimum at $s=0$. Next we write $\left(\|\cdot\|_{2}\right.$ denotes the $L^{2}\left(\Omega ; \mathbb{R}^{3}\right)$ norm $)$

$$
\begin{aligned}
\int_{\Omega}|X(t, g(s, x))-x|^{2} \mathrm{~d} x & =\int_{\Omega}|X(t, x)-g(-s, x)|^{2} \mathrm{~d} x \\
& =\|X(t, \cdot)\|_{2}^{2}+\|\mathrm{Id}\|_{2}^{2}-2 \int_{\Omega} X(t, x) \cdot g(-s, x) \mathrm{d} x
\end{aligned}
$$


to see that minimizing $i$ amounts to maximizing

$$
j(s):=\int_{\Omega} X(t, x) \cdot g(-s, x) \mathrm{d} x .
$$

(Note that this problem makes sense even for $X(t, \cdot) \in L^{1}\left(\Omega ; \mathbb{R}^{d}\right)$.) Imposing that the first variation be zero at $s=0$ for all $\mathbf{v} \in \mathcal{V}(\Omega)$ amounts to

$$
\int_{\Omega} X(t, x) \cdot \mathbf{v}(x) \mathrm{d} x=0 \text { for all } \mathbf{v} \in \mathcal{V}(\Omega)
$$

which holds because $X(t, \cdot)$ is the gradient of a $W^{1,1}(\Omega)$ map. A non-positive second variation of $j$ at $s=0$ yields (2.3) via the calculations

$$
\begin{aligned}
j^{\prime \prime}(s)=\frac{\mathrm{d}}{\mathrm{d} s} j^{\prime}(s) & =-\frac{\mathrm{d}}{\mathrm{d} s} \int_{\Omega} X(t, x) \cdot \mathbf{v}(g(-s, x)) \mathrm{d} x \\
& =\int_{\Omega} X(t, x) \cdot \nabla \mathbf{v}(g(-s, x)) \mathbf{v}(g(-s, x)) \mathrm{d} x
\end{aligned}
$$

the condition $g(0, x)=x$ for all $x \in \Omega$, and integration by parts.

What if we have better regularity for $P$ ? In view of $\nabla[X \cdot \mathbf{v}]=(\nabla X)^{T} \mathbf{v}+(\nabla \mathbf{v})^{T} X$ and $\nabla \cdot \mathbf{v} \equiv 0$ in $\Omega$, we have

$$
0=\int_{\Omega}(\nabla X(t, x))^{T} \mathbf{v}(x) \cdot \mathbf{v}(x) \mathrm{d} x+\int_{\Omega}(\nabla \mathbf{v})^{T}(x) X(t, x) \cdot \mathbf{v}(x) \mathrm{d} x,
$$

which reveals

$$
\begin{aligned}
\int_{\Omega} P(t, x) \nabla \cdot[\nabla \mathbf{v}(x) \mathbf{v}(x)] \mathrm{d} x & =-\int_{\Omega} X(t, x) \cdot \nabla \mathbf{v}(x) \mathbf{v}(x) \mathrm{d} x \\
& =-\int_{\Omega}[\nabla \mathbf{v}(x)]^{T} X(t, x) \cdot \mathbf{v}(x) \mathrm{d} x \\
& =\int_{\Omega} \nabla X(t, x) \mathbf{v}(x) \cdot \mathbf{v}(x) \mathrm{d} x,
\end{aligned}
$$

due to the fact that $\nabla X=\nabla^{2} P$ is symmetric. The following corollary arises as an immediate consequence of Theorem 2.2:

Corollary 2.3. Let $(P, \mathbf{u})$ be a conservative (i.e., $E(t)=E(0)$ for all $t \geq 0$ ) distributional solution for (1.2) which satisfies $P(t, \cdot) \in W^{2,1}(\Omega)$ for all $t \geq 0$. Assume that for any $t>0$ and $\mathbf{v} \in \mathcal{V}(\Omega)$, the function $J[t$, $\mathbf{v}]$ has a minimum at $s=0$ over the interval $(-t, \infty)$. Then

$$
\int_{\Omega} \nabla X(t, x) \mathbf{v}(x) \cdot \mathbf{v}(x) \mathrm{d} x \geq 0 \text { for all } t \geq 0 \text { and all } \mathbf{v} \in \mathcal{V}(\Omega)
$$

Remark 2.4. The proof of Theorem 2.2 shows that (2.5) arises as a consequence of $s=0$ being a local minimizer for $i$ defined in (2.4). In turn, this is a consequence of $s=0$ minimizing $J[t, \mathbf{v}]$ over $(-t, \infty)$. 


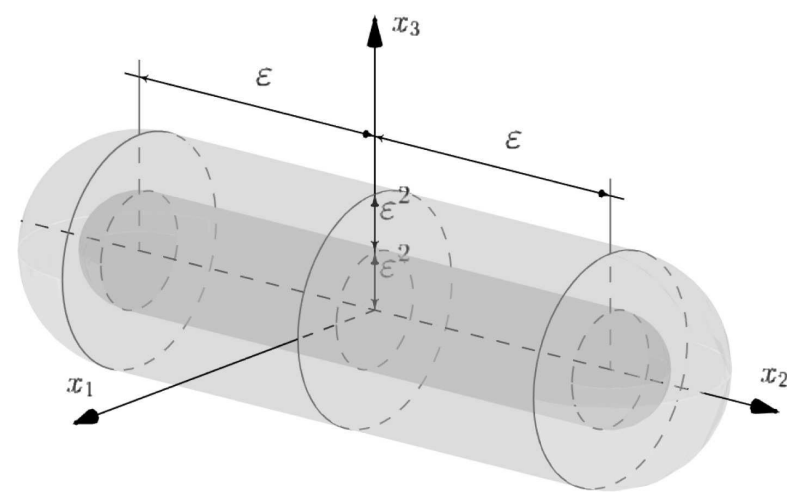

FiguRE 1. Region $D_{\varepsilon}^{2}$ for $d=3$.

\subsection{The convexity of the modified pressure}

Here we show how (2.3) implies the convexity of the modified pressure potential $P(t, \cdot)$.

Theorem 2.5. Let $d \geq 2$ be an integer, $\Omega \subset \mathbb{R}^{d}$ be open and $\Phi \in L^{1}(\Omega)$. Then

$$
\int_{\Omega} \Phi(x) \nabla \cdot[\nabla \mathbf{v}(x) \mathbf{v}(x)] \mathrm{d} x \geq 0 \text { for all } \mathbf{v} \in \mathcal{V}(\Omega)
$$

if and only if $\Phi$ is convex in $\Omega$.

Proof. 1. We first assume $\Phi \in C^{3}(\bar{\Omega})$ so that, as seen in Corollary 2.3, (2.6) is equivalent to

$$
\int_{\Omega} M(x) \mathbf{v}(x) \cdot \mathbf{v}(x) \mathrm{d} x \geq 0 \text { for all } \mathbf{v} \in \mathcal{V}(\Omega),
$$

where $M(x):=\nabla^{2} \Phi(x)$. This is obvious if $\Phi$ is convex, so we focus on proving the converse. Let $\zeta \in C_{c}^{1}(1,2)$ and $\varepsilon>0$. For each $1 \leq k \leq d$ consider the domain $D_{\varepsilon}^{k}$ consisting of the region between the cylinders $\varepsilon^{2} \leq \mid x-$ $x_{k} \mathbf{e}_{k} \mid \leq 2 \varepsilon^{2}$ for $-\varepsilon \leq x_{k} \leq \varepsilon$, capped at $x_{k}=-\varepsilon$ by the spherical shell between the hemispheres $\left|x+\varepsilon \mathbf{e}_{k}\right|=\varepsilon^{2}$ and $\left|x+\varepsilon \mathbf{e}_{k}\right|=2 \varepsilon^{2}$ for $-\varepsilon-2 \varepsilon^{2} \leq x_{k} \leq-\varepsilon$, and at $x_{k}=\varepsilon$ by the spherical shell between the hemispheres $\left|x-\varepsilon \mathbf{e}_{k}\right|=\varepsilon^{2}$ and $\left|x-\varepsilon \mathbf{e}_{k}\right|=2 \varepsilon^{2}$ for $\varepsilon+2 \varepsilon^{2} \geq x_{k} \geq \varepsilon$; here, $\mathbf{e}_{k}$ denotes the $k^{\text {th }}$ column of the $d \times d$ identity matrix (the region $D_{\varepsilon}^{2}$ for $d=3$ is shown in Fig. 1). Consider the vector fields

$$
\mathbf{v}_{\varepsilon}^{1}(x)=\left\{\begin{array}{l}
-x_{2} \zeta\left(\varepsilon^{-2}\left|x-x_{1} \mathbf{e}_{1}\right|\right) \mathbf{e}_{1} \text { if }\left|x_{1}\right| \leq \varepsilon \\
\zeta\left(-\varepsilon^{-2}\left|x+\varepsilon \mathbf{e}_{1}\right|\right)\left[-x_{2} \mathbf{e}_{1}+\left(x_{1}+\varepsilon\right) \mathbf{e}_{2}\right] \text { if } x_{1}<-\varepsilon \\
\zeta\left(-\varepsilon^{-2}\left|x-\varepsilon \mathbf{e}_{1}\right|\right)\left[-x_{2} \mathbf{e}_{1}+\left(x_{1}-\varepsilon\right) \mathbf{e}_{2}\right] \text { if } x_{1}>\varepsilon
\end{array}\right.
$$

and

$$
\mathbf{v}_{\varepsilon}^{k}(x)=\left\{\begin{array}{l}
-x_{1} \zeta\left(\varepsilon^{-2}\left|x-x_{k} \mathbf{e}_{k}\right|\right) \mathbf{e}_{1} \text { if }\left|x_{k}\right| \leq \varepsilon \\
\zeta\left(-\varepsilon^{-2}\left|x+\varepsilon \mathbf{e}_{k}\right|\right)\left[-x_{1} \mathbf{e}_{k}+\left(x_{k}+\varepsilon\right) \mathbf{e}_{1}\right] \text { if } x_{1}<-\varepsilon \\
\zeta\left(-\varepsilon^{-2}\left|x-\varepsilon \mathbf{e}_{k}\right|\right)\left[-x_{1} \mathbf{e}_{k}+\left(x_{k}-\varepsilon\right) \mathbf{e}_{1}\right] \text { if } x_{1}>\varepsilon
\end{array}\right.
$$

if $2 \leq k \leq d$.

We have:

(i) $\mathbf{v}_{\varepsilon}^{k} \in C_{c}\left(D_{\varepsilon}^{k} ; \mathbb{R}^{d}\right)$ for all $\varepsilon>0$ and all $1 \leq k \leq d$. 
(ii) $\mathbf{v}_{\varepsilon}^{k}$ is $C^{1}$ in the cylindrical shell and in each of the spherical shells. Furthermore, $\nabla \cdot \mathbf{v}_{\varepsilon}^{k}=0$ in each of these subdomains.

Note that (i) and (ii) readily imply that $\nabla \cdot \mathbf{v}_{\varepsilon}^{k}=0$ in $D_{\varepsilon}^{k}$ in the sense of distributions. Since it is continuous and has compact support in $D_{\varepsilon}^{k}, \mathbf{v}_{\varepsilon}^{k}$ can be approximated uniformly by smooth, compactly supported, divergence-free vector fields (may use convolutions with mollifiers from a smooth, compactly supported kernel). This means that if a matrix-valued function $M: \Omega \rightarrow \mathcal{M}_{d}(\mathbb{R})$ (the latter notation is for the vector space of $d \times d$ real matrices) lies in $L^{1}$ and satisfies (2.7), then it will satisfy this for all $\mathbf{v} \in C_{c}\left(\Omega ; \mathbb{R}^{3}\right)$ with zero distributional divergence. In particular, if we fix $x_{0} \in \Omega$, then we can take $\mathbf{v}(x):=Q_{0}^{T} \mathbf{v}_{\varepsilon}^{k}\left(Q_{0}\left(x-x_{0}\right)\right)$, where $x_{0} \in \Omega$ and $r_{0}>0$ and $\varepsilon>0$ are sufficiently small so that $x_{0}+D_{\varepsilon}^{k} \subset B\left(x_{0}, r_{0}\right) \subset \Omega$, and $Q_{0}$ is a constant orthogonal matrix. Let $B_{0}:=B\left(0, r_{0}\right)$. Since $B_{0}$ is invariant under the transformation $x \rightarrow Q_{0} x$, we deduce

$$
\int_{B_{0}} Q_{0} M\left(x_{0}+Q_{0}^{T} x\right) Q_{0}^{T} \mathbf{v}_{\varepsilon}^{k}(x) \cdot \mathbf{v}_{\varepsilon}^{k}(x) \mathrm{d} x=\int_{\Omega} M(x) Q_{0}^{T} \mathbf{v}_{\varepsilon}^{k}\left(Q_{0}\left(x-x_{0}\right)\right) \cdot Q_{0}^{T} \mathbf{v}_{\varepsilon}^{k}\left(Q_{0}\left(x-x_{0}\right)\right) \mathrm{d} x \geq 0,
$$

which means (due to $\left.\operatorname{spt}\left(\mathbf{v}_{\varepsilon}^{k}\right) \subset D_{\varepsilon}^{k} \subset B_{0}\right)$

$$
\int_{D_{\varepsilon}^{k}} P(x) \mathbf{v}_{\varepsilon}^{k}(x) \cdot \mathbf{v}_{\varepsilon}^{k}(x) \mathrm{d} x \geq 0
$$

where $P(x):=Q_{0} M\left(x_{0}+Q_{0}^{T} x\right) Q_{0}^{T}$. Since $M\left(x_{0}\right)$ is symmetric, let $Q_{0}$ be the orthogonal matrix such that $Q_{0} M\left(x_{0}\right) Q_{0}^{T}=P(0)$ is diagonal. Since $M \in C^{1}\left(\bar{\Omega} ; \mathcal{M}_{d}(\mathbb{R})\right)$, there exists a real constant $C$ such that $\mid P(x)-$ $P(0)|\leq C| x \mid$ for all $x \in B_{0}$. In particular, $|P(x)-P(0)| \leq C \varepsilon$ for all $x \in D_{\varepsilon}^{k}$ (where the constant may be different but we do not update the notation). Thus,

$$
\left|\int_{D_{\varepsilon}^{k}} P(x) \mathbf{v}_{\varepsilon}^{k}(x) \cdot \mathbf{v}_{\varepsilon}^{k}(x) \mathrm{d} x-\int_{D_{\varepsilon}^{k}} P(0) \mathbf{v}_{\varepsilon}^{k}(x) \cdot \mathbf{v}_{\varepsilon}^{k}(x) \mathrm{d} x\right| \leq C \varepsilon \int_{D_{\varepsilon}^{k}}\left|\mathbf{v}_{\varepsilon}^{k}(x)\right|^{2} \mathrm{~d} x \leq C \varepsilon^{2 d+4},
$$

where we took into account that $\mathcal{L}^{d}\left(D_{\varepsilon}^{k}\right)=O\left(\varepsilon^{2 d-1}\right)$ and $\left|\mathbf{v}_{\varepsilon}^{k}\right|=O\left(\varepsilon^{2}\right)$. So

$$
\int_{D_{\varepsilon}^{k}} P(0) \mathbf{v}_{\varepsilon}^{k}(x) \cdot \mathbf{v}_{\varepsilon}^{k}(x) \mathrm{d} x \geq-C \varepsilon^{2 d+4} .
$$

The volume of each spherical shell is $O\left(\varepsilon^{2 d}\right)$, so

$$
\left|\int_{S_{\varepsilon}^{k}} P(0) \mathbf{v}_{\varepsilon}^{k}(x) \cdot \mathbf{v}_{\varepsilon}^{k}(x) \mathrm{d} x\right| \leq C \varepsilon^{2 d+4},
$$

where $S_{\varepsilon}^{k}$ is the union of the two spherical shells. Since $D_{\varepsilon}^{k} \backslash S_{\varepsilon}^{k}=: C_{\varepsilon}^{k}$ is the cylindrical shell of equations $-\varepsilon \leq x_{k} \leq \varepsilon, \varepsilon^{2} \leq\left|x-x_{k} \mathbf{e}_{k}\right| \leq 2 \varepsilon^{2}$, we conclude

$$
\int_{-\varepsilon}^{\varepsilon}\left[\int_{A_{\varepsilon}^{k}} P(0) \mathbf{v}_{\varepsilon}^{k}(x) \cdot \mathbf{v}_{\varepsilon}^{k}(x) d \bar{x}_{k}\right] \mathrm{d} x_{k}=\int_{C_{\varepsilon}^{k}} P(0) \mathbf{v}_{\varepsilon}^{k}(x) \cdot \mathbf{v}_{\varepsilon}^{k}(x) \mathrm{d} x \geq-C \varepsilon^{2 d+4}
$$

where and $\bar{x}_{k}:=\left(x_{1}, \ldots, x_{k-1}, x_{k+1}, \ldots, x_{d}\right)$ and $A_{\varepsilon}^{k} \subset \mathbb{R}^{d-1}$ is the annulus $\varepsilon^{2} \leq\left|\bar{x}_{k}\right|=\left|x-x_{k} \mathbf{e}_{k}\right| \leq 2 \varepsilon^{2}$ (naturally, if $k=1$ or $k=d, \bar{x}_{k}$ is defined similarly). Let $k=1$. Then (2.10) becomes

$$
2 \varepsilon P_{11}(0) \int_{A_{\varepsilon}^{1}} x_{2}^{2} \zeta^{2}\left(\varepsilon^{-2}\left|\bar{x}_{1}\right|\right) d \bar{x}_{1} \geq-C \varepsilon^{2 d+4},
$$


which, upon changing variables $\left(y_{2}, \ldots, y_{d}\right)=\bar{y}_{1}=\varepsilon^{-2} \bar{x}_{1}$ and letting $A^{1}:=\left\{\bar{y}_{1} \in \mathbb{R}^{d-1}: 1 \leq\left|\bar{y}_{1}\right| \leq 2\right\}$, yields

$$
2 \varepsilon^{2 d+3} P_{11}(0) \int_{A^{1}} y_{2}^{2} \zeta^{2}\left(\left|\bar{y}_{1}\right|\right) d \bar{y}_{1} \geq-C \varepsilon^{2 d+4} .
$$

Since this holds for all sufficiently small $\varepsilon>0$, it suffices to take $\zeta$ not identically zero to conclude $P_{11}(0) \geq 0$. Likewise, if we take $2 \leq k \leq d$ in (2.10), we get $P_{k k}(0) \geq 0$. But $P_{k k}(0)$ is precisely the $k^{\text {th }}$ eigenvalue of $M\left(x_{0}\right)$, so we are done.

2. Next we drop the $C^{3}$ up to the boundary assumption and deal with the general case, i.e. $\Phi \in L^{1}(\Omega)$. For $\varepsilon>0$ sufficiently small we denote $\Omega_{\varepsilon}:=\{x \in \Omega: \operatorname{dist}(x, \partial \Omega)>\varepsilon\}$ and let $\eta^{\varepsilon}$ be the standard mollifier supported in $B(0, \varepsilon)$. Define on $\Omega_{\varepsilon}$ the function $\Phi^{\varepsilon}:=\eta^{\varepsilon} * \Phi$. Take $\mathbf{v} \in \mathcal{V}(\Omega)$ supported in $\Omega_{\varepsilon}$ and compute

$$
\begin{aligned}
\int_{\Omega_{\varepsilon}} \Phi^{\varepsilon} \nabla \cdot[(\nabla \mathbf{v}) \mathbf{v}] \mathrm{d} x & =\int_{\Omega_{\varepsilon}}\left[\int_{B(x, \varepsilon)} \eta^{\varepsilon}(x-y) \Phi(y) \mathrm{d} y\right] \nabla \cdot[\nabla \mathbf{v}(x) \mathbf{v}(x)] \mathrm{d} x \\
& =\int_{\Omega_{\varepsilon}}\left[\int_{B(0, \varepsilon)} \eta^{\varepsilon}(y) \Phi(x-y) \mathrm{d} y\right] \nabla \cdot[\nabla \mathbf{v}(x) \mathbf{v}(x)] \mathrm{d} x \\
& =\int_{B(0, \varepsilon)} \eta^{\varepsilon}(y)\left[\int_{\Omega_{\varepsilon}-y} \Phi(z) \nabla \cdot[\nabla \mathbf{v}(z+y) \mathbf{v}(z+y)] \mathrm{d} z\right] \mathrm{d} y .
\end{aligned}
$$

But $\mathbf{v}(\cdot+y) \in \mathcal{V}(\Omega)$ is supported in $\Omega_{\varepsilon}-y \subset \Omega$ if $\mathbf{v}$ is supported in $\Omega_{\varepsilon}$, so the inner integral is nonnegative by (2.6) for all $y \in B(0, \varepsilon)$ and all $\mathbf{v} \in \mathcal{V}(\Omega)$ supported in $\Omega_{\varepsilon}$. It follows that $\Phi^{\varepsilon}$ is (according to the proof above for the regular case) convex on $\Omega_{\varepsilon}$. But $\Phi^{\varepsilon}$ converges a.e. to $\Phi$ in $\Omega$, so $\Phi$ is convex.

Conversely, if $\Phi$ is convex, then $\Phi^{\varepsilon}$ is convex on $\Omega_{\varepsilon}$ and so

$$
\int_{\Omega} \Phi \nabla \cdot[(\nabla \mathbf{v}) \mathbf{v}] \mathrm{d} x=\lim _{\varepsilon \rightarrow 0^{+}} \int_{\Omega_{\varepsilon}} \Phi^{\varepsilon} \nabla \cdot[(\nabla \mathbf{v}) \mathbf{v}] \mathrm{d} x=\lim _{\varepsilon \rightarrow 0^{+}} \int_{\Omega_{\varepsilon}} \nabla^{2} \Phi^{\varepsilon} \mathbf{v} \cdot \mathbf{v} \mathrm{d} x \geq 0
$$

which finishes the proof.

\section{Connection With optimal transport AND Open PROBlems}

\subsection{Optimal transport with quadratic cost}

As seen in the proof of Theorem 2.2, the condition that $J[t, \mathbf{v}]$ have a local minimum at 0 implies that

$$
E[t, \mathbf{v}](s):=\int_{\Omega}|X(t, g[\mathbf{v}](s, x))-x|^{2} \mathrm{~d} x
$$

has a local minimum at 0 for all $\mathbf{v} \in \mathcal{V}(\Omega)$ and all $t \geq 0$, i.e. $X(t, \cdot)$ minimizes the energy with respect to measurepreserving variations induced by one-parameter families of flow maps of compactly supported, divergence-free vector fields. The Optimal Transport theory tells us that for any Borel probability density $\rho$ with finite second moment and any $X \in L^{2}\left(\rho ; \mathbb{R}^{\mathrm{d}}\right)$ we have

$$
\int_{\Omega}|X(x)-x|^{2} \rho(x) \mathrm{d} x=\min _{Y \sim X} \int_{\Omega}|Y(x)-x|^{2} \rho(x) \mathrm{d} x
$$

if and only if $X$ is the gradient of a convex function, where $Y \sim X$ means

$$
\int_{\Omega} \zeta(Y(x)) \rho(x) \mathrm{d} x=\int_{\Omega} \zeta(X(x)) \rho(x) \mathrm{d} x \text { for all } \zeta \in C_{b}\left(\mathbb{R}^{\mathrm{d}}\right) .
$$


Furthermore, if we denote by $\mu:=X_{\#} \rho, \mathcal{P}(A)$ the set of Borel probabilities on $A$, and

$$
\Gamma(\rho, \mu):=\left\{\gamma \in \mathcal{P}\left(\mathbb{R}^{\mathrm{d}} \times \mathbb{R}^{\mathrm{d}}\right): \operatorname{proj}_{1} \gamma=\rho, \operatorname{proj}_{2} \gamma=\mu\right\}
$$

we have that the square of the quadratic Wasserstein distance between $\rho$ and $\mu$ is

$$
W_{2}^{2}(\rho, \mu)=\int_{\mathbb{R}^{\mathrm{d}}}|X(x)-x|^{2} \rho(x) \mathrm{d} x=\min _{\gamma \in \Gamma(\rho, \mu)} \iint_{\mathbb{R}^{\mathrm{d}} \times \mathbb{R}^{\mathrm{d}}}|x-y|^{2} \gamma(\mathrm{d} x, \mathrm{~d} y)
$$

and $\bar{\gamma}:=(\operatorname{Id} \times X)_{\#} \rho$ is the unique minimizer of the right hand side.

I. If $\rho=\left.\mathcal{L}^{d}\right|_{\Omega}$, without any extra assumptions on $X$ (see [4]), if $d>1$ it is not true that any $Y \sim X$ can be written as $Y=X \circ \mathbf{s}$ for some Borel map $\mathbf{s}$ that preserves the Lebesgue measure restricted to $\Omega$. Even more exclusive is the set of maps $Y$ of the form $Y=X \circ g$ for some measure-preserving, invertible $g$. And it is not known whether all such maps can be approximated by flow maps $g[\mathbf{v}](s, \cdot)$ for some $\mathbf{v} \in \mathcal{V}(\Omega)$ and $s \in \mathbb{R}$. Yet, Proposition 3.1 below shows that minimizing the energy over each of these measure-preserving variations is equivalent. More precisely, let

$$
\begin{gathered}
\mathcal{S}(\Omega):=\left\{\mathbf{s}: \Omega \rightarrow \Omega \text { Borel }: \mathbf{s}_{\#} \rho=\rho\right\}, \\
\mathcal{G}(\Omega):=\left\{g: \Omega \rightarrow \Omega \text { Borel, invertible }: g_{\#} \rho=\rho\right\}
\end{gathered}
$$

and

$$
\mathcal{M}(X):=\left\{Y: \Omega \rightarrow \mathbb{R}^{\mathrm{d}} \text { Borel }: Y_{\#} \rho=X_{\#} \rho\right\}
$$

Proposition 3.1. Let $X \in L^{2}\left(\Omega ; \mathbb{R}^{\mathrm{d}}\right)$ be a Borel map. The following are equivalent:

(i) $s=0$ locally minimizes $\int_{\Omega}|X(g[\mathbf{v}](s, x))-x|^{2} \mathrm{~d} x$ for all $\mathbf{v} \in \mathcal{V}(\Omega)$.

(ii) $\operatorname{Id}_{\Omega}$ minimizes $\int_{\Omega}|X(g(x))-x|^{2} \mathrm{~d} x$ over all $g \in \mathcal{G}(\Omega)$.

(iii) $\operatorname{Id}_{\Omega}$ minimizes $\int_{\Omega}|X(\mathbf{s}(x))-x|^{2} \mathrm{~d} x$ over all $\mathbf{s} \in \mathcal{S}(\Omega)$.

(iv) $X$ minimizes $\int_{\Omega}|Y(x)-x|^{2} \mathrm{~d} x$ over all $Y \in \mathcal{M}(X)$.

$(v)\left(\operatorname{Id}_{\Omega} \times X\right)_{\#} \rho$ minimizes $\iint_{\mathbb{R}^{\mathrm{d}} \times \mathbb{R}^{\mathrm{d}}}|x-y|^{2} \gamma(\mathrm{d} x, \mathrm{~d} y)$ over all $\gamma \in \Gamma\left(\rho, X_{\#} \rho\right)$.

(vi) There exists $\Phi \in H^{1}(\Omega)$ convex such that $X=\nabla \Phi$ a.e. in $\Omega$.

Proof. The equivalence of $(i v),(v),(v i)$ follows from the Monge-Kantorovich theory with cost $c(x, y)=|x-y|^{2}$. There is a proposition in [6] (Lemma 6.4) which ensures that any $Y \in \mathcal{M}(X)$ can be approximated uniformly by maps of the type $X \circ g$ with $g \in \mathcal{G}(\Omega)$, so $(i v)$ and $(i i)$ are equivalent. Same proposition applied to $X$ and $X \circ \mathbf{s}$ for any $\mathbf{s} \in \mathcal{S}(\Omega)$ shows that the latter can be approximated uniformly by maps $X \circ g$ for $g \in \mathcal{G}(\Omega)$ (since $X \circ \mathbf{s} \in \mathcal{M}(X)$ ). Thus, $(i i)$ and $(i i i)$ are equivalent. It is easy to see that $(i i)$ implies $(i)$. Finally, by Theorem 2.2 , (i) implies $(v i)$.

II. If $\rho \in C^{1}(\bar{\Omega})$ is positive, we consider

$$
\mathcal{V}[\rho](\Omega):=\left\{\mathbf{w} \in C_{c}^{1}\left(\Omega ; \mathbb{R}^{\mathrm{d}}\right): \nabla \cdot(\rho \mathbf{w})=0 \text { in } \Omega\right\}
$$


The flow $g[\mathbf{w}]$ of a vector field $\mathbf{w} \in \mathcal{V}[\rho](\Omega)$ is defined by

$$
\partial_{s} g(s, x)=\mathbf{w}(g(s, x)) \text { in } \mathbb{R} \times \Omega, g(0, x)=x \text { in } \Omega .
$$

The proof of Proposition A.1 can be modified (see Rem. A.2) to show that $g(s, \cdot)$ is a diffeomorphism of $\Omega$ which satisfies both $g(s, \cdot)^{-1}=g(-s, \cdot)$ and $g(s, \cdot)_{\#} \rho=\rho$ for all $s \in \mathbb{R}$. Indeed, the first claim is a simple consequence of the semigroup property for an autonomous ODE. As for the second, we use the initial value problem that $\mathrm{d}(s, x):=\operatorname{det}\left[\nabla_{x} g(s, x)\right]$ satisfies, namely

$$
\partial_{s} d(s, x)=(\nabla \cdot \mathbf{w})(g(s, x)) \mathrm{d}(s, x), \quad \mathrm{d}(0, x)=1 .
$$

Since $g(s, \cdot)$ is a diffeomorphism of $\Omega$ and the above IVP shows $d$ is always positive, all we need to prove is that

$$
\rho(g(s, x)) \mathrm{d}(s, x)=\rho(x) \text { for all } s \in \mathbb{R}, x \in \Omega .
$$

Since $\mathrm{d}(0, \cdot)=1$ and $g(0, \cdot)=\mathrm{Id}$, it is enough to show that the derivative with respect to $s$ of the left hand side vanishes at any $s \in \mathbb{R}$. To that effect we compute

$$
\begin{aligned}
\partial_{s}[(\rho \circ g) d](s, x) & =\nabla \rho(g(s, x)) \cdot \partial_{s} g(s, x) \mathrm{d}(s, x)+\rho(g(s, x)) \partial_{s} \mathrm{~d}(s, x) \\
& =[\nabla \rho(g(s, x)) \cdot \mathbf{w}(g(s, x))+\rho(g(s, x))(\nabla \cdot \mathbf{w})(g(s, x))] \mathrm{d}(s, x) \\
& =[\nabla \cdot(\rho \mathbf{w})](g(s, x)) \mathrm{d}(s, x)=0,
\end{aligned}
$$

so the claim that $g(s, \cdot)_{\#} \rho=\rho$ is proved. Thus, if $s=0$ maximizes

$$
j(s):=\int_{\Omega} X(x) \cdot g[\mathbf{w}](s, x) \rho(x) \mathrm{d} x
$$

we infer

$$
\int_{\Omega} X(x) \cdot \mathbf{w}(x) \rho(x) \mathrm{d} x=0 \text { and } \int_{\Omega} X(x) \cdot \nabla \mathbf{w}(x) \mathbf{w}(x) \rho(x) \mathrm{d} x \leq 0 .
$$

Since any $\mathbf{v} \in \mathcal{V}(\Omega)$ can be written as $\rho \mathbf{w}$ for some $\mathbf{w} \in \mathcal{V}[\rho](\Omega)$ in a unique way (for any $\mathbf{v} \in \mathcal{V}(\Omega), \mathbf{w}=\mathbf{v} / \rho)$, from the first variation we deduce $X$ must be a gradient in $\Omega$. If we assume $\Phi \in W^{2,1}(\Omega)$, i.e. $X \in W^{1,1}\left(\Omega ; \mathbb{R}^{\mathrm{d}}\right)$, and use $\nabla[X \cdot \mathbf{w}]=(\nabla X)^{T} \mathbf{w}+(\nabla \mathbf{w})^{T} X$ and integrate against $\rho \mathbf{w}$ on $\Omega$ we get

$$
0 \leq-\int_{\Omega} X(x) \cdot \nabla \mathbf{w}(x) \mathbf{w}(x) \rho(x) \mathrm{d} x=\int_{\Omega} \nabla X(x) \mathbf{w}(x) \cdot \mathbf{w}(x) \rho(x) \mathrm{d} x .
$$

We deduce

$$
\int_{\Omega}[\rho(x)]^{-1} \nabla X(x) \mathbf{v}(x) \cdot \mathbf{v}(x) \mathrm{d} x \geq 0 \text { for all } \mathbf{v} \in \mathcal{V}(\Omega),
$$

which yields, as before, the nonnegative definiteness of $[\rho(x)]^{-1} \nabla X(x)$ for all $x \in \Omega$. Since $\rho>0$ in $\Omega$, this is equivalent to $\nabla X(x)$ being nonnegative definite at all $x \in \Omega$. If the assumption $\Phi \in W^{2,1}(\Omega)$ is relaxed to $\Phi \in W^{1,1}(\Omega)$, it is not clear to us how to finish the argument. If we denote $\alpha:=1 / \rho$, we obviously have $\alpha \in C^{1}(\bar{\Omega})$ and $\alpha>0$ on $\bar{\Omega}$ if and only if $\rho \in C^{1}(\bar{\Omega})$ and $\rho>0$ on $\bar{\Omega}$. Thus, we can reformulate the problem as: 
let $\Phi \in W^{1,1}(\Omega)$ and $\rho \in C^{1}(\bar{\Omega})$ such that $\rho>0$ on $\bar{\Omega}$. Then $\Phi$ is convex if and only if

$$
\int_{\Omega} \nabla \Phi \cdot \nabla(\rho \mathbf{v}) \mathbf{v} \mathrm{d} x \leq 0 \text { for all } \mathbf{v} \in \mathcal{V}(\Omega)
$$

As seen above, this is settled if $\Phi \in W^{2,1}(\Omega)$; we have not been able to use a convolution argument as in the last part of the proof of Theorem 2.2 to accommodate the less regular case. Of course, integration by parts in the last displayed inequality shows that the problem can be pushed further to:

Conjecture 3.2. Let $\Phi \in L^{1}(\Omega)$. Then the following are equivalent:

(i) $\Phi$ is convex;

(ii) There exists $\rho \in C^{1}(\bar{\Omega})$ such that $\rho>0$ on $\bar{\Omega}$ and

$$
\int_{\Omega} \Phi \nabla \cdot[\nabla(\rho \mathbf{v}) \mathbf{v}] \mathrm{d} x \geq 0 \text { for all } \mathbf{v} \in \mathcal{V}(\Omega)
$$

(iii) (3.4) holds for all $\rho \in C^{1}(\bar{\Omega})$ such that $\rho>0$ on $\bar{\Omega}$ and all $\mathbf{v} \in \mathcal{V}(\Omega)$.

If $\rho \equiv$ const $\neq 0$ or if $\Phi \in W^{2,1}(\Omega)$, this is settled above, but the general case is impeded by $\rho$ not being translation invariant (essential to the last part of the proof of Theorem 2.2, where $\left.\rho \equiv \mathcal{L}^{d}\right|_{\Omega}$ ).

III. If $\rho_{0}$ is nonnegative and is only assumed to lie in $L^{1}(\Omega)$ we can only make sense of $\nabla \cdot\left(\rho_{0} \mathbf{v}\right)=0$ in the sense of distributions, and the computations we performed above to prove that $g(s, \cdot)_{\#} \rho_{0}=\rho_{0}$ for any $s \in \mathbb{R}$ do not apply, due to the lack of regularity for $\rho_{0}$. We define

$$
\mathcal{V}\left[\rho_{0}\right](\Omega):=\left\{\mathbf{v} \in C_{c}^{1}\left(\Omega ; \mathbb{R}^{\mathrm{d}}\right): \nabla \cdot\left(\rho_{0} \mathbf{v}\right)=0 \text { as a distribution in } \Omega\right\} .
$$

Once again, we take $g$ to be the flow of $\mathbf{v} \in \mathcal{V}\left[\rho_{0}\right](\Omega)$ originating at the identity map; let $\rho(s, \cdot):=g(s, \cdot)_{\#} \rho_{0}$, so that $\rho_{0}=\rho(0, \cdot)$. We would like to prove that $\rho(s, \cdot)=\rho_{0}$ for all $s \in \mathbb{R}$. Let $\zeta \in C_{c}^{1}\left(\mathbb{R}^{\mathrm{d}}\right)$ and compute

$$
\begin{aligned}
\frac{\mathrm{d}}{\mathrm{d} s} \int_{\Omega} \zeta(x) \rho(s, \mathrm{~d} x) & =\int_{\Omega} \nabla \zeta(g(s, x)) \cdot \mathbf{v}(g(s, x)) \rho_{0}(x) \mathrm{d} x \\
& =\int_{\Omega} \nabla \zeta(x) \cdot \mathbf{v}(x) \rho(s, \mathrm{~d} x),
\end{aligned}
$$

i.e. $\rho(s, x)$ is a distributional solution of the initial value problem

$$
\partial_{s} \rho+\nabla \cdot(\rho \mathbf{v})=0 \text { in }(0, \infty) \times \Omega, \quad \rho(0, \cdot)=\rho_{0} \text { in } \Omega .
$$

Let us denote by $\tilde{\mathbf{v}}$ the extension of $\mathbf{v}$ by zero outside $\Omega$ and let $\tilde{g}$ be its flow originating at $\operatorname{Id}_{\mathbb{R}^{\mathrm{d}}}$. It is easy to see that $\tilde{g}(s, \cdot)=g(s, \cdot)$ in $\Omega$ and $\tilde{g}(s, \cdot)=$ Id outside $\Omega$. If we denote by $\tilde{\rho}_{0}$ and $\tilde{\rho}(s, \cdot)$ the extensions by zero outside $\Omega$ of $\rho_{0}$ and $\rho(s, \cdot)$ respectively, we consequently have

$$
\tilde{\rho}(s, x)=\tilde{\rho}_{0}(\tilde{g}(-s, x)) \operatorname{det}\left[\nabla_{x} \tilde{g}(-s, x)\right] \text { for all } s \in \mathbb{R}, x \in \mathbb{R}^{\mathrm{d}},
$$

i.e. $\tilde{g}(s, \cdot)_{\#} \tilde{\rho}_{0}=\tilde{\rho}(s, \cdot)$ for all $s \in \mathbb{R}$. We deduce $\tilde{\rho}$ solves

$$
\partial_{s} \tilde{\rho}+\nabla \cdot(\tilde{\rho} \tilde{\mathbf{v}})=0 \text { in }(0, \infty) \times \mathbb{R}^{\mathrm{d}}, \quad \tilde{\rho}(0, \cdot)=\tilde{\rho}_{0} \text { in } \mathbb{R}^{\mathrm{d}}
$$

in the sense of distributions. Since $\nabla \cdot\left(\rho_{0} \mathbf{v}\right)=0$ in $\Omega$, it is easy to see that $\tilde{\rho}_{0}$ is also a solution (stationary). We may now use Theorem 6.1.1 [8], which guarantees that uniqueness for the PDE above is equivalent to uniqueness 
for the ODE

$$
\partial_{s} \alpha(s, x)=\tilde{\mathbf{v}}(\alpha(s, x)), \quad \alpha(0, x)=\tilde{\rho}_{0}(x)
$$

for any $x \in \Omega$, provided that $\tilde{\mu}_{0}\left(\mathbb{R}^{\mathrm{d}} \backslash \Omega\right)=0$ (where $\tilde{\mu}_{0}$ is the measure on $\mathbb{R}^{\mathrm{d}}$ whose density is $\tilde{\rho}_{0}$ ), which holds by the definition of $\tilde{\rho}_{0}$. But this ODE does have a unique solution for every $x \in \Omega$, namely $g(\cdot, x)$, so we conclude $\tilde{\rho}(s, \cdot)=\tilde{\rho}_{0}$ in $\mathbb{R}^{\mathrm{d}}$, i.e. $\rho(s, \cdot)=\rho_{0}$ in $\Omega$ for all $s \in \mathbb{R}$. Thus, $g(s, \cdot)_{\#} \rho_{0}=\rho_{0}$ for all $s \in \mathbb{R}$, which means that we can now conclude, as in part II above, that

$$
\int_{\Omega} \nabla X(x) \mathbf{v}(x) \cdot \mathbf{v}(x) \rho_{0}(x) \mathrm{d} x \geq 0 \text { for all } \mathbf{v} \in \mathcal{V}\left[\rho_{0}\right] .
$$

Since $\rho_{0}$ is not necessarily sufficiently regular and positive in $\Omega$, we cannot finish the argument as in part II $\left(\rho_{0} \mathbf{v}\right.$ may not exhaust all the vector fields in $\mathcal{V}(\Omega)$ ). We leave it open to see if the last displayed inequality does imply that $\nabla X$ is nonnegative definite on the support of $\rho_{0}$.

\section{2. $1 \mathrm{D}$ case}

Since $\mathcal{V}(\Omega)$ consists only of the null function if $d=1$, there is no $1 \mathrm{D}$ version of the above arguments, i.e. one cannot use flow maps of compactly supported divergence free vector fields to prove that optimal maps are gradients of convex functions. As a workaround, we use the $d=2$ case in the following way: let us assume $X \in L^{2}(0,1)$ is the optimal map pushing $\chi:=\left.\mathcal{L}^{1}\right|_{(0,1)}$ forward to some probability measure $\rho$, i.e.

$$
W_{2}^{2}(\chi, \rho)=\int_{0}^{1}|X(x)-x|^{2} \mathrm{~d} x .
$$

First notice that $S(x, y):=(X(x), X(y))$ pushes $\chi \otimes \chi$ forward to $\rho \otimes \rho$ and so

$$
W_{2}^{2}(\chi \otimes \chi, \rho \otimes \rho) \leq \int_{0}^{1} \int_{0}^{1}|(X(x), X(y))-(x, y)|^{2} \mathrm{~d} x \mathrm{~d} y=2 W_{2}^{2}(\chi, \rho) .
$$

On the other hand, if $\gamma$ is a transport plan between $\chi \otimes \chi$ and $\rho \otimes \rho$, i.e. a Borel probability measure on $\mathbb{R}^{4}$ such that

$$
\int_{\mathbb{R}^{4}} \xi\left(x_{1}\right) \zeta\left(x_{2}\right) \gamma\left(\mathrm{d} x_{1}, \mathrm{~d} x_{2}, \mathrm{~d} y_{1}, \mathrm{~d} y_{2}\right)=\int_{0}^{1} \xi\left(x_{1}\right) \mathrm{d} x_{1} \int_{0}^{1} \zeta\left(x_{2}\right) \mathrm{d} x_{2}
$$

and

$$
\int_{\mathbb{R}^{4}} \xi\left(y_{1}\right) \zeta\left(y_{2}\right) \gamma\left(\mathrm{d} x_{1}, \mathrm{~d} x_{2}, \mathrm{~d} y_{1}, \mathrm{~d} y_{2}\right)=\int_{\mathbb{R}} \xi\left(y_{1}\right) \rho\left(\mathrm{d} y_{1}\right) \int_{\mathbb{R}} \zeta\left(y_{2}\right) \rho\left(\mathrm{d} y_{2}\right)
$$

for all continuous and of at most quadratic growth $\xi$ and $\zeta$, then the measures $\tilde{\gamma}_{i}$ defined by

$$
\int_{\mathbb{R}^{2}} \zeta\left(x_{i}, y_{i}\right) \tilde{\gamma}_{i}\left(\mathrm{~d} x_{i}, \mathrm{~d} y_{i}\right)=\int_{\mathbb{R}^{4}} \zeta\left(x_{i}, y_{i}\right) \gamma\left(\mathrm{d} x_{1}, \mathrm{~d} x_{2}, \mathrm{~d} y_{1}, \mathrm{~d} y_{2}\right), \quad i=1,2,
$$

are both transport plans between $\chi$ and $\rho$. Since

$$
\int_{\mathbb{R}^{4}}\left|\left(x_{1}, x_{2}\right)-\left(y_{1}, y_{2}\right)\right|^{2} \gamma\left(\mathrm{d} x_{1}, \mathrm{~d} x_{2}, \mathrm{~d} y_{1}, \mathrm{~d} y_{2}\right)=\sum_{i=1}^{2} \int_{\mathbb{R}^{2}}\left|x_{i}-y_{i}\right|^{2} \tilde{\gamma}_{i}\left(\mathrm{~d} x_{i}, \mathrm{~d} y_{i}\right),
$$


we select $\gamma$ optimal in the left hand side to conclude

$$
W_{2}^{2}(\chi \otimes \chi, \rho \otimes \rho) \geq 2 W_{2}^{2}(\chi, \rho) .
$$

Thus, we have equality in (3.6), which implies $S$ is an optimal map pushing $\chi \otimes \chi$ forward to $\rho \otimes \rho$. As we have seen above, this means $S=\nabla \Phi$ for some convex map $\Phi$, i.e. $X(x)=\partial_{x} \Phi(x, y), X(y)=\partial_{y} \Phi(x, y)$ for some convex $\Phi$. Of course, this means $\Phi(x, y)=\phi(x)+\phi(y)$ for some convex $\phi$, and so $X=\phi^{\prime}$ a.e. in $(0,1)$.

\section{APPENDIX A.}

Proposition A.1. Let $\Omega \subset \mathbb{R}^{\mathrm{d}}$ be open and $C^{1}$ and let $\mathbf{v} \in C^{1}\left(\mathbb{R} \times \bar{\Omega} ; \mathbb{R}^{\mathrm{d}}\right)$ such that $\nabla_{x} \cdot \mathbf{v} \equiv 0$ in $\mathbb{R} \times \Omega$ and $\mathbf{v} \cdot \nu \equiv 0$ on $\mathbb{R} \times \partial \Omega$. Then there exists a unique flow of measure-preserving diffeormorphisms for $\mathbf{v}$ in $\bar{\Omega}$. More precisely, for every $x \in \bar{\Omega}$ the solution of

$$
\partial_{s} g(s, x)=\mathbf{v}(s, g(s, x)), \quad g(0, x)=x
$$

exists and is unique for all $s \in \mathbb{R}$. Furthermore, the map $x \rightarrow g(s, x)$ is a measure-preserving $C^{1}$-diffeomorphism of $\bar{\Omega}$. Moreover, if $\mathbf{v}$ does not depend on $s$, then $[g(s, \cdot)]^{-1}=g(-s, \cdot)$ for all $s \in \mathbb{R}$.

Proof. We divide the proof in three parts.

1. First we show that for any $x \in \Omega$, the solution $g(s, x)$ exists for all $s \in \mathbb{R}$, is unique, and stays in $\Omega$ for all time (observe that $\mathbf{v}$ is not even defined outside $\bar{\Omega}$ ). Fix $x \in \Omega$. By the theorems of existence, uniqueness and continuation of solutions for ODE, note that the solution exists and is unique for as long as $g(s, x) \in \bar{\Omega}$. Thus, it is enough to prove that there is no time $s \in \mathbb{R}$ such that $g(s, x) \in \partial \Omega$. Assume there is such $s_{0}>0$ when the solution first reaches $\partial \Omega$, i.e. $g(s, x) \in \Omega$ for all $s \in\left[0, s_{0}\right)$ and $g\left(s_{0}, x\right)=y^{0} \in \partial \Omega$. Since $\partial \Omega \in C^{1}$, we know there exists a function $f \in C^{1}\left(\mathbb{R}^{d-1}\right)$ such that locally around $y^{0}$ the boundary $\partial \Omega$ is given by the surface of equation $y_{d}=f\left(y_{1}, \ldots, y_{d-1}\right)$. If $z \in \mathbb{R}^{\mathrm{d}}$, denote $\tilde{z}=\left(z_{1}, \ldots, z_{d-1}\right)$. Consider the unique local (i.e. $s \in\left(s_{0}-\delta, s_{0}+\delta\right)$ for some small $\delta>0)$ solution $\left(u_{1}, \ldots, u_{d-1}\right)$ for the ODE system

$$
\dot{u}_{k}(t)=v_{k}\left(t, u_{1}, \ldots, u_{d-1}, f\left(u_{1}, \ldots, u_{d-1}\right)\right), \quad u_{k}\left(t_{0}\right)=y_{k}^{0}, \quad k=1, \ldots, d-1 .
$$

Define $u_{d}:\left(s_{0}-\delta, s_{0}+\delta\right) \rightarrow \mathbb{R}$ by $u_{d}(s):=f\left(u_{1}(s), \ldots, u_{d-1}(s)\right)$ and note that, if we set $\mathbf{u}:=\left(u_{1}, \ldots, u_{d-1}, u_{d}\right)$ and differentiate, we find

$$
\dot{u}_{d}=\nabla f(\tilde{\mathbf{u}}) \cdot \tilde{\mathbf{v}}(s, \mathbf{u}) .
$$

Since

$$
\mathbf{u}(s) \in \partial \Omega \text { for all } s \in\left(s_{0}-\delta, s_{0}+\delta\right)
$$

(by taking $\delta>0$ even smaller, if necessary), the previous equation implies

$$
\dot{u}_{d}(s)=v_{d}(s, \mathbf{u}(s)) \text {. }
$$

Here we have used that on the portion of the boundary $\partial \Omega$ around $y^{0}$ described by the equation $y_{d}=f(\tilde{y})$ we have that the normal direction to $\partial \Omega$ at $y$ is $(\nabla f(\tilde{y}),-1) \in \mathbb{R}^{\mathrm{d}}$, and so, by hypothesis,

$$
\nabla f(\tilde{y}) \cdot \tilde{\mathbf{v}}(s, y)-v_{d}(s, y)=0 .
$$


According to (A.2) and (A.4), we have constructed a solution of

$$
\dot{\mathbf{u}}=\mathbf{v}(s, \mathbf{u}), \quad \mathbf{u}\left(s_{0}\right)=y^{0},
$$

which is defined and unique on $\left(s_{0}-\delta, s_{0}+\delta\right)$ for some $\delta>0$ sufficiently small. However, we know that $g(s, x)$ satisfies the same system of ODE's on $\left(s_{0}-\delta, s_{0}\right]$ and $g\left(s_{0}, x\right)=y^{0}$. By uniqueness, we infer $g(s, x)=\mathbf{u}(s)$ for all $s \in\left(s_{0}-\delta, s_{0}\right]$, which, by (A.3), contradicts the fact that $s_{0}>0$ is the earliest time when $g(\cdot, x)$ reaches $\partial \Omega$. The case $s_{0}<0$ can be treated similarly. We conclude that the unique solution $g(\cdot, x)$ is defined on the whole $\mathbb{R}$ and satisfies $g(\mathbb{R}, x) \subset \Omega$ for any $x \in \Omega$, i.e. $g(\mathbb{R}, \Omega) \subset \Omega$. A straightforward application of Gronwall's Lemma to the variation of constants formula yields

$$
|g(s, x+h)-g(s, x)| \leq|h| e^{s\|\nabla \mathbf{v}\|_{\infty}}=: c(s)|h|,
$$

so $g(s, \cdot)$ is Lipschitz for every $s \in \mathbb{R}$. It follows that $g(s, \cdot)$ can be extended by continuity up to $\partial \Omega$; since $g(s, \Omega) \subset \Omega$, we also infer $g(s, \bar{\Omega}) \subset \bar{\Omega}$. We retain the notation $g(s, \cdot)$ for this extension. From

$$
g(s, x)=x+\int_{0}^{s} \mathbf{v}(\sigma, g(\sigma, x)) \mathrm{d} \sigma \text { for all }(s, x) \in \mathbb{R} \times \Omega
$$

the continuous extension proved above shows that for every $x \in \bar{\Omega}$ the solution of (A.1) exists and is unique for all $s \in \mathbb{R}$. We next claim that $g(s, \partial \Omega) \subset \partial \Omega$ for all $s \in \mathbb{R}$; otherwise we would have some $\Omega \ni y_{0}=g\left(s_{0}, x_{0}\right)$ for some $s_{0} \in \mathbb{R}$ and $x_{0} \in \partial \Omega$. But then $\tilde{g}(s, x):=g\left(s+s_{0}, x\right)$ solves $\partial_{s} \tilde{g}\left(s, x_{0}\right)=\mathbf{v}_{s_{0}}\left(t, \tilde{g}\left(s, x_{0}\right)\right)$ with $\tilde{g}\left(0, x_{0}\right)=y_{0}$, where $\mathbf{v}_{s_{0}}(s, x):=\mathbf{v}\left(s+s_{0}, x\right)$. However, $\tilde{g}\left(-s_{0}, x_{0}\right)=x_{0} \in \partial \Omega$, so we obtain the same contradiction as above (for $\mathbf{v}_{s_{0}}$, which also satisfies $\mathbf{v}_{s_{0}} \cdot \nu=0$ on $\partial \Omega$ ). So,

$$
g(s, \Omega) \subset \Omega \text { and } g(s, \partial \Omega) \subset \partial \Omega \text { for all } s \in \mathbb{R} .
$$

2. Next we show that $g(s, \cdot) \in C^{1}\left(\Omega ; \mathbb{R}^{\mathrm{d}}\right)$ for all $s \in \mathbb{R}$. Fix $x \in \bar{\Omega}$ and $h \in \mathbb{R}^{\mathrm{d}}$ with $|h|$ sufficiently small so that $x+h \in \Omega$. Since, $\mathbf{v} \in C^{1}\left(\bar{\Omega} ; \mathbb{R}^{\mathrm{d}}\right)$, we have

$$
\lim _{|p| \rightarrow 0} \frac{1}{|p|}|\mathbf{v}(y+p)-\mathbf{v}(y)-\nabla \mathbf{v}(y) p|=0 \text { uniformly w.r.t. } y \in \bar{\Omega} .
$$

Now, (A.5) and (A.7) imply

$$
\begin{aligned}
\mid \mathbf{v}(g(s, x+h))-\mathbf{v}(g(s, x)) & -\nabla \mathbf{v}(g(s, x))[g(s, x+h)-g(s, x)] \mid \\
= & A(s, x, h)=|h| O(|h|)
\end{aligned}
$$

uniformly for $x \in \bar{\Omega}$ and for $s$ in compact sets, where $O:[0, \infty) \rightarrow[0, \infty)$ is continuous at the origin and $O(0)=0$. Next, for each $x \in \bar{\Omega}$, let us consider the matrix solution to

$$
\dot{M}(s, x)=\nabla \mathbf{v}(g(s, x)) M(s, x), \quad M(0, x)=I_{d},
$$

where $I_{d}$ is the $d \times d$ identity matrix. Set, again for $h \in \mathbb{R}^{\mathrm{d}}$ with $|h|$ sufficiently small, $w:=w(s, x, h)=M(s, x) h$ to see that it satisfies

$$
\dot{w}(s, x, h)=\nabla \mathbf{v}(g(s, x)) w(s, x, h), \quad w(0, x, h)=h .
$$


We employ again the variation of constants formula to deduce

$$
\begin{aligned}
B(s, x, h): & =|g(s, x+h)-g(s, x)-w(s, x, h)| \\
& \leq \int_{0}^{s}|\mathbf{v}(g(\sigma, x+h))-\mathbf{v}(g(\sigma, x))-w(\sigma, x, h)| \mathrm{d} \sigma \\
& \leq \int_{0}^{s} A(\sigma, x, h) \mathrm{d} \sigma+\|\nabla \mathbf{v}\|_{\infty} \int_{0}^{s} B(\sigma, x, h) \mathrm{d} \sigma .
\end{aligned}
$$

By (A.8) and Gronwall's Lemma we get

$$
|B(s, x, h)|=o(|h|) \text { uniformly for } x \in \bar{\Omega} \text { and } s \text { in compact sets, }
$$

i.e. $g(s, \cdot)$ is differentiable at $x \in \bar{\Omega}$ uniformly for $s$ in compact sets. Furthermore, we obtain $\nabla g(s, x)=M(s, x)$, which implies (by (A.9)) that the map $x \rightarrow \nabla g(s, x)$ is continuous in $\bar{\Omega}$ (uniformly for $s$ in compact sets). Thus, $g(s, \cdot) \in C^{1}\left(\bar{\Omega} ; \mathbb{R}^{\mathrm{d}}\right)$ for all $s \in \mathbb{R}$.

3. Finally, we prove that for all $s \in \mathbb{R}$ the map $g(s, \cdot)$ is a measure-preserving $C^{1}$ diffeomorphism of $\Omega$. By the uniqueness theorem for the prescribed-value problem at any $s_{0} \in \mathbb{R}$ we infer that $g(s, \cdot)$ is one-to-one for all $s \in \mathbb{R}$. Let $\Omega_{s}:=g(s, \Omega)$ so that $g(s, \cdot)$ is a one-to-one map from $\Omega$ onto $\Omega_{s}$. By direct computation we arrive at an IVP for an ODE satisfied by $\mathrm{d}(s, x):=\operatorname{det} \nabla_{x} g(s, x)$, namely

$$
\partial_{s} d(s, x)=\nabla \cdot \mathbf{v}(s, g(s, x)) \mathrm{d}(s, x), \quad \mathrm{d}(0, x)=1,
$$

which implies that the Jacobian of $g(s, x)$ has determinant equal to one for all $s \in \mathbb{R}$ and all $x \in \Omega$. Thus,

$$
\int_{\Omega} \zeta(g(s, x)) \mathrm{d} x=\int_{\Omega_{s}} \zeta(y) \mathrm{d} y \text { for all } \zeta \in C\left(\mathbb{R}^{\mathrm{d}}\right) .
$$

First, by choosing $\zeta \equiv 1$ we infer that $\mathcal{L}^{d}\left(\Omega_{s}\right)=\mathcal{L}^{d}(\Omega)$. Assume $\Omega \backslash \Omega_{s_{0}} \neq \emptyset$ for some $s_{0} \in \mathbb{R}$ and let $y_{0} \in \Omega \backslash \Omega_{s_{0}}$. Since $\Omega_{s_{0}} \subset \Omega$ (by (A.6)) and $\mathcal{L}^{d}\left(\Omega_{s}\right)=\mathcal{L}^{d}(\Omega)$, we deduce that there is no ball centered at $y_{0}$ that is contained in $\Omega \backslash \Omega_{s_{0}}$, so there exists a sequence $\left\{x_{n}\right\}_{n} \subset \Omega$ such that $g\left(s_{0}, x_{n}\right)$ converges to $y_{0}$. Let $x_{0}$ be an accumulation point for the sequence $\left\{x_{n}\right\}_{n}$; then $g\left(s_{0}, x_{0}\right)=y_{0}$ by the continuity of $g\left(s_{0}, \cdot\right)$. Since $y_{0} \in \Omega \backslash \Omega_{s_{0}}$, it must be that $x_{0} \in \partial \Omega$, but that contradicts $g\left(s_{0}, \partial \Omega\right) \cap \Omega=\emptyset$ (see (A.6)). It follows that $\Omega_{s_{0}}=\Omega$. Since $s_{0}$ is arbitrary, we conclude $g(s, \cdot)$ is a $C^{1}$ diffeomorphism of $\bar{\Omega}$, which, by (A.12), is also measure preserving.

Remark A.2. If $\mathbf{v}$ is time-independent, then the assumption $\nabla \cdot \mathbf{v} \equiv 0$ is not necessary to conclude that $g(s, \cdot)$ is a $C^{1}$ diffeomorphism of $\Omega$. Indeed, again by uniqueness, we infer (the semigroup property) that if $\mathbf{v}$ is time-independent, then $g(s+t, x)=g(s, g(t, x)$ ), which implies $g(s, \cdot)$ (whose domain is $\Omega$ ) is invertible, with $[g(s, \cdot)]^{-1}=g(-s, \cdot)$ (whose domain is also $\Omega$ ) for all $s \in \mathbb{R}$. If, in addition, $\nabla \cdot \mathbf{v} \equiv 0$ in $\Omega$, we conclude as above that the $C^{1}$-diffeomorphisms $g(s, \cdot)$ are volume preserving.

Proposition A.3. Let $d \geq 2$ be integer and let $B$ denote the open unit ball in $\mathbb{R}^{d}$. Then:

(i) The quantity $\zeta \rightarrow\left\|\partial_{x_{i}} \zeta\right\|_{L^{2}(B)}$ defines a norm on $H_{0}^{1}(B)$ for $i=1, \ldots, d$.

(ii) None of the norms from (i) is metrically equivalent to the standard Euclidean $H^{1}$-norm on $H_{0}^{1}(B)$.

Proof. Let us, without loss of generality, fix $i=1$. (i) All the conditions from the definition of a norm are trivial to check, including the positive definiteness: indeed, let $\zeta \in C_{c}^{\infty}(B)$ and extend it by zero outside the ball. For $x \in B$ we have

$$
\zeta(x)=\int_{-1}^{x_{1}} \partial_{x_{1}} \zeta\left(y, x_{2}, \ldots, x_{d}\right) \mathrm{d} y
$$


so if $\partial_{x_{1}} \zeta \equiv 0$ in $B$ we get $\zeta \equiv 0$.

(ii) If $d=2$ take $u_{n}(x)=f_{1}\left(x_{1}\right) g_{n}\left(x_{2}\right)$, where both $f_{1}$ and $g_{n}$ are nonzero, supported in $(-0.5,0.5)$ and smooth. Then $u_{n} \in H_{0}^{1}(B)$. Assume $g_{n}$ converges uniformly to $g\left(x_{2}\right)=\sqrt{0.25-x_{2}^{2}}$ (extended by 0 outside $(-0.5,0.5)$ ); existence of such sequence $g_{n}$ is obvious. So $u_{n}$ converges uniformly to $u(x)=f_{1}\left(x_{1}\right) g\left(x_{2}\right)$, while $\partial_{x_{1}} u_{n}$ converges uniformly to $f_{1}^{\prime}\left(x_{1}\right) g\left(x_{2}\right)$. Both $u$ and $\partial_{x_{1}} u$ are in $L^{2}(B)$, while $\partial_{x_{2}} u$ is not. This shows that the $L^{2}$-norm of $\partial_{x_{1}} u$ is not complete on $H_{0}^{1}(B)$, so it is not equivalent to the standard $H_{0}^{1}(B)$ norm. If $d \geq 3$ we multiply $u_{n}$ by a nonzero compactly supported smooth function $f_{2}\left(x_{3}, \ldots, x_{d}\right)$ supported in $(-0.5,0.5)^{d-2}$ and we conclude similarly.

Proposition A.4. Let $B$ denote the open unit ball in $\mathbb{R}^{3}$. Then:

(i) The quantity $(P, Q, R)=: \mathbf{u} \rightarrow\left\|P_{z}-R_{x}\right\|_{L^{2}(B)}+\left\|Q_{x}-P_{y}\right\|_{L^{2}(B)}$ defines a norm on $\mathrm{d}_{0}$.

(ii) The norm from (i) is not metrically equivalent to the standard Euclidean $H^{1}$-norm on $\mathrm{d}_{0}$.

Proof. For (i) the only thing that needs checking is that $\tilde{P}_{z}=\tilde{R}_{x}$ and $\tilde{Q}_{x}=\tilde{P}_{y}$ imply $\mathbf{u}=(\tilde{P}, \tilde{Q}, \tilde{R}) \equiv 0$ in $B$. So take $(P, Q, R)$ in $C_{c}^{2}\left(B ; \mathbb{R}^{3}\right)$ such that $\tilde{P}=R_{y}-Q_{z}, \tilde{Q}=P_{z}-R_{x}, \tilde{R}=Q_{x}-P_{y}$. We have that $\tilde{P}_{z}=\tilde{R}_{x}$ and $\tilde{Q}_{x}=\tilde{P}_{y}$ imply

$$
R_{y z}-Q_{z z}=Q_{x x}-P_{x y} \text { and } P_{x z}-R_{x x}=R_{y y}-Q_{y z}
$$

or, equivalently,

$$
\Delta Q=\partial_{y} \nabla \cdot(P, Q, R) \text { and } \Delta R=\partial_{z} \nabla \cdot(P, Q, R)
$$

Thus, $\Delta Q_{z}=\Delta R_{y}$ and, since $Q_{z} \equiv R_{y}=0$ on $\partial B$, we deduce $0=R_{y}-Q_{z}=\tilde{P}$ in $B$; so now (A.13) imply $P_{x y}-Q_{x x}=P_{x z}-R_{x x}=0$, i.e. $\tilde{Q}$ and $\tilde{R}$ are independent of $x$. Due to their compact support in $B$, we infer $\tilde{Q} \equiv \tilde{R} \equiv 0$ in $B$.

(ii) Let $Q \equiv R \equiv 0$ and for each positive integer $n$ we define $P_{n}(x, y, z):=u(x) v(n y, n z)$, where $u, v$ are smooth, nonzero and compactly supported in $(-0.5,0.5)$ and $(-0.5,0.5)^{2}$, respectively; it follows that $P_{n}$ is smooth, nonzero and compactly supported in $B$. Let $\left(\tilde{P}_{n}, \tilde{Q}_{n}, \tilde{R}_{n}\right)=\nabla \times\left(P_{n}, 0,0\right)=\left(0, P_{n, z},-P_{n, y}\right)$, so that

$$
\mathbf{u}_{n}:=\nabla \times\left(\tilde{P}_{n}, \tilde{Q}_{n}, \tilde{R}_{n}\right)=\left(-P_{n, y y}-P_{n, z z},-P_{n, x y}, P_{n, x z}\right) .
$$

Since

$$
\begin{aligned}
\left\|P_{n, y y}+P_{n, z z}\right\|_{L^{2}(B)}^{2} & =n^{4} \int_{-0.5}^{0.5} u^{2}(x) \mathrm{d} x \int_{(-0.5,0.5)^{2}}|\Delta v(n y, n z)|^{2} \mathrm{~d} y \mathrm{~d} z \\
& =n^{2} \int_{-0.5}^{0.5} u^{2}(x) \mathrm{d} x \int_{(-0.5,0.5)^{2}}|\Delta v(y, z)|^{2} \mathrm{~d} y \mathrm{~d} z
\end{aligned}
$$

and

$$
\begin{aligned}
\left\|P_{n, x y}\right\|_{L^{2}(B)}^{2}+\left\|P_{n, x z}\right\|_{L^{2}(B)}^{2} & =n^{2} \int_{-0.5}^{0.5} u^{2}(x) \mathrm{d} x \int_{(-0.5,0.5)^{2}}|\nabla v(n y, n z)|^{2} \mathrm{~d} y \mathrm{~d} z \\
& =\int_{-0.5}^{0.5}\left|u^{\prime}(x)\right|^{2} \mathrm{~d} x \int_{(-0.5,0.5)^{2}}|\nabla v(y, z)|^{2} \mathrm{~d} y \mathrm{~d} z
\end{aligned}
$$

it is enough to notice that $v$ is non-harmonic (as a non-identically vanishing, compactly supported smooth function) to obtain the desired thesis. 


\section{A.1 The 2D and $3 \mathrm{D}$ cases revisited}

Here we present different proofs that (2.3) is equivalent to the convexity of $P(t, \cdot)$ as a function of $x \in \Omega$ if $d=2,3$; this is achieved by means of Propositions A.5 and A.6, which are not consequences of Theorem 2.5, but imply it if $d=2,3$. Albeit more complex, they are, in some sense, more straight forward than the proof in Section 2 as no explicitly constructed test functions (vector fields such as $\mathbf{v}_{\varepsilon}^{k}$ ) need be involved. Furthermore, we believe they are worth presenting here, as they rely on a spatially locally continuous decomposition of the symmetric matrix $\nabla^{2} P(t, x)$ as $Q^{T}(t, x) \Lambda(t, x) Q(t, x)$ for each fixed $t$, where $Q$ is orthogonal and $\Lambda$ is diagonal. Whereas the continuity of the eigenvalues with respect to the matrix entries is a well-known fact, it is not always true that $Q$ can be chosen in such a way that it varies continuously with respect to the factored matrix [19]. What turns out to be of tremendous help is the simple observation that any nonnegative definite perturbation of $\nabla^{2} P$ satisfies $(2.5)$ if $\nabla^{2} P$ does. It then becomes a matter of carefully choosing arbitrarily small appropriate perturbations $\nabla^{2} S_{\varepsilon}$ of $\nabla^{2} P$ for which $\nabla^{2}\left(P+S_{\varepsilon}\right)$ does admit a continuous $Q_{\varepsilon}^{T} \Lambda_{\varepsilon} Q_{\varepsilon}$-decomposition in a neighborhood of any given point in $\Omega$. This continuity will enable us to prove that the eigenvalues of $\nabla^{2}\left(P+S_{\varepsilon}\right)$ are nonnegative, which, by the continuity of the eigenvalues with respect to the matrix entries, implies by letting $\varepsilon \rightarrow 0^{+}$that the eigenvalues of $\nabla^{2} P$ are nonnegative. In what follows, the entries of the matrix $M$ are denoted by $m_{i j}$, while the vector space of all $d \times d$ real symmetric matrices is denoted by $\mathcal{S}_{d}(\mathbb{R})$.

\section{A.1.12D case}

Here we show that if $d=2$, then (2.3) is equivalent to the convexity of $P(t, \cdot)$. Note that the existence of a sequence $\left\{\zeta_{n}\right\}_{n}$ with the properties below is given (up to the transformation $x \rightarrow x_{0}+Q_{0} J r_{0}\left(x-x_{0}\right)$ ) in the proof of Proposition A.3.

Proposition A.5. Let $x_{0} \in \mathbb{R}^{2}, r_{0}>0$ and $M: B_{0}:=B\left(x_{0}, r_{0}\right) \rightarrow \mathcal{S}_{2}(\mathbb{R})$ be continuous. Denote by $Q_{0}$ the constant orthogonal matrix such that $Q_{0} M\left(x_{0}\right) Q_{0}^{T}$ is diagonal. Let $\left\{\zeta_{n}\right\}_{n} \in C_{c}^{\infty}\left(B_{0}\right)$ be such that

$$
\operatorname{spt}\left(\zeta_{n}\right) \subset B\left(x_{0}, r_{n}\right) \text { for a decreasing sequence }\left\{r_{n}\right\}_{n} \downarrow 0
$$

and

$$
\frac{\left(Q_{0} J \nabla \zeta_{n}\right)_{2}}{\left\|\zeta_{n}\right\|_{H^{1}\left(B_{0}\right)}} \rightarrow 0 \text { as } n \rightarrow \infty
$$

If

$$
\int_{B_{0}} J M(x) J \nabla \zeta_{n}(x) \cdot \nabla \zeta_{n}(x) \mathrm{d} x \leq 0 \text { for all } n \geq 1
$$

then the first eigenvalue of $M\left(x_{0}\right)$ is nonnegative.

Proof. Let $\Lambda(x)$ be the $2 \times 2$ diagonal matrix with real diagonal entries $\lambda_{1}(x), \lambda_{2}(x)$ (eigenvalues of $M(x)$ ). By the well-known fact that roots of polynomials vary continuously with respect to their coefficients, the continuity of $M$ implies that $\Lambda$ is continuous on $\Omega$.

1. Assume first that $\alpha_{0}:=m_{12}\left(x_{0}\right) \neq 0$. If necessary, restrict $r_{0}$ closer to zero such that for all $x \in B\left(x_{0}, r_{0}\right)$ we have $m_{12}(x) \geq \alpha_{0} / 2$ if $\alpha_{0}>0$, or $m_{12}(x) \leq \alpha_{0} / 2$ if $\alpha_{0}<0$. Assume $\lambda_{1}\left(x_{0}\right)=:-2 \lambda^{-}<0$.

We claim that there exists a continuous function $\overline{B\left(x_{0}, r\right)} \ni x \rightarrow Q(x) \in \mathcal{M}_{2}(\mathbb{R})$ such that $Q(x)$ is orthogonal and

$$
M(x)=Q^{T}(x) \Lambda(x) Q(x) \text { for all } x \in \overline{B\left(x_{0}, r_{0}\right)}
$$


Indeed, since $m_{12}(x)$ is bounded away from zero uniformly with respect to $x \in \overline{B\left(x_{0}, r_{0}\right)}$, we see that the two eigenvalues are distinct for any such $x$ and we can calculate that an orthonormal pair of eigenvectors corresponding to $\lambda_{1}(x)$ and $\lambda_{2}(x)$, respectively, consists of

$$
\left(\begin{array}{c}
\frac{m_{12}(x)}{\sqrt{m_{12}^{2}(x)+\left[m_{11}(x)-\lambda_{1}(x)\right]^{2}}} \\
\frac{\lambda_{1}(x)-m_{11}(x)}{\sqrt{\left.m_{12}^{2}(x)+m_{11}(x)-\lambda_{1}(x)\right]^{2}}}
\end{array}\right) \text { and }\left(\begin{array}{c}
\frac{m_{12}(x)}{\sqrt{m_{12}^{2}(x)+\left[m_{11}(x)-\lambda_{2}(x)\right]^{2}}} \\
\frac{\lambda_{2}(x)-m_{11}(x)}{\sqrt{m_{12}^{2}(x)+\left[m_{11}(x)-\lambda_{2}(x)\right]^{2}}}
\end{array}\right) \text {. }
$$

Since $m_{12}$ is bounded away from zero on $\overline{B\left(x_{0}, r\right)}$, we easily see that these two vectors are continuous as functions of $x$ on $\overline{B\left(x_{0}, r\right)}$. We choose $Q(x)$ to be the matrix whose columns are these two vectors (in this order), so the claim is proved. We may ocasionally drop the dependence on $x$ to unburden notation; we have

$$
M \mathbf{v} \cdot \mathbf{v}=(Q \mathbf{v})^{T} \Lambda(Q \mathbf{v})=\lambda_{1}\left|(Q \mathbf{v})_{1}\right|^{2}+\lambda_{2}\left|(Q \mathbf{v})_{2}\right|^{2} .
$$

If necessary, restrict $r$ even further such that $\lambda_{1}(x)<-\lambda^{-}$for all $x \in B\left(x_{0}, r\right)$. Let $n$ be sufficiently large such that $\zeta_{n} \in C_{c}^{1}\left(B\left(x_{0}, r\right)\right)$ and put $\mathbf{v}_{n}:=J \nabla \zeta_{n}$. We conclude that

$$
\begin{aligned}
\int_{\Omega} M(x) \mathbf{v}_{n}(x) \cdot \mathbf{v}_{n}(x) \mathrm{d} x & =\int_{B\left(x_{0}, r\right)} M(x) \mathbf{v}_{n}(x) \cdot \mathbf{v}_{n}(x) \mathrm{d} x \\
& \leq-\lambda^{-} \int_{B\left(x_{0}, r\right)}\left|\left(Q \mathbf{v}_{n}\right)_{1}\right|^{2} \mathrm{~d} x+\lambda^{+} \int_{B\left(x_{0}, r\right)}\left|\left(Q \mathbf{v}_{n}\right)_{2}\right|^{2} \mathrm{~d} x
\end{aligned}
$$

where

$$
\lambda^{+}:=\max _{x \in \overline{B\left(x_{0}, r\right)}}\left|\lambda_{2}(x)\right| .
$$

We now employ (A.16) to infer

$$
\int_{B\left(x_{0}, r\right)}\left|\nabla \zeta_{n}\right|^{2} \mathrm{~d} x \leq \bar{\lambda} \int_{B\left(x_{0}, r\right)}\left|\left(Q J \nabla \zeta_{n}\right)_{2}\right|^{2} \mathrm{~d} x \text { for all sufficiently large } n,
$$

where $\bar{\lambda}:=\left(\lambda^{-}+\lambda^{+}\right) / \lambda^{-}$. Note that we have used $|Q \mathbf{v}|=|\mathbf{v}|$ in $B\left(x_{0}, r\right)$ because $Q(x)$ is an orthogonal matrix for all $x \in B\left(x_{0}, r\right)$. Let $Q_{0}:=Q\left(x_{0}\right)$ and for all $0<s \leq r$ we set (since $Q-Q_{0}$ is continuous on the closure of $\left.B\left(x_{0}, r\right)\right)$

$$
\left\|Q-Q_{0}\right\|_{L^{\infty}\left(B\left(x_{0}, s\right)\right)}=: \eta_{s}<\infty
$$

so that $\left|\left(Q(x) \mathbf{v}_{n}(x)\right)_{2}-\left(Q_{0} \mathbf{v}_{n}(x)\right)_{2}\right| \leq \eta_{s}\left|\mathbf{v}_{n}(x)\right|$ for all $x \in B\left(x_{0}, s\right)$ and all sufficiently large $n$ such that $\zeta_{n} \in C_{c}^{1}\left(B\left(x_{0}, s\right)\right)$. Thus,

$$
\left|\left(Q(x) \mathbf{v}_{n}(x)\right)_{2}\right|^{2} \leq 2\left[\left|\left(Q_{0} \mathbf{v}_{n}(x)\right)_{2}\right|^{2}+\eta_{s}^{2}\left|\mathbf{v}_{n}(x)\right|^{2}\right]
$$

Since $\eta_{s} \rightarrow 0^{+}$as $s \rightarrow 0^{+}$, there exists $0<s_{0} \leq r$ such that $0<2 \bar{\lambda} \eta_{s_{0}}^{2}<1$; (A.17) then implies

$$
\int_{B\left(x_{0}, s_{0}\right)}\left|\nabla \zeta_{n}\right|^{2} \mathrm{~d} x \leq \lambda_{0} \int_{B\left(x_{0}, s_{0}\right)}\left|\left(Q_{0} J \nabla \zeta_{n}\right)_{2}\right|^{2} \mathrm{~d} x \text { for all sufficiently large } n,
$$


where $\lambda_{0}:=2 \bar{\lambda} /\left(1-2 \bar{\lambda} \eta_{s_{0}}^{2}\right)$. This contradicts the properties of the sequence $\left\{\zeta_{n}\right\}_{n}$.

2 . Now assume $m_{12}\left(x_{0}\right)=0$. Let $\delta>0$ and define $\Phi_{\delta}(x):=\delta\left(x_{1}+x_{2}\right)^{2} / 2$. Obviously, $M+\nabla^{2} \Phi_{\delta}$ satisfies (A.16) and, since $\partial_{12} \Phi_{\delta}\left(x_{0}\right)=\delta>0$, we are back under the restrictions of part 1 of this proof, so the eigenvalue $\lambda_{1}^{\delta}\left(x_{0}\right)$ (first eigenvalue of $M\left(x_{0}\right)+\nabla^{2} \Phi_{\delta}\left(x_{0}\right)$ ) is nonnegative. Once again, the eigenvalues are continuous functions of the matrix entries, so we let $\delta \rightarrow 0^{+}$to infer $\lambda_{1}\left(x_{0}\right) \geq 0$.

\section{A.1.2 The 3D case}

The $d=3$ case is slightly more delicate because compactly supported divergence free vector fields have a more complex structure. There is also significantly more work involved in showing that we can always reduce the problem to one where we locally have a decomposition $\nabla^{2} \Phi=Q^{T} \Lambda Q$ such that $Q$ is a continuous function of $x$. The proof is no different from the $2 \mathrm{D}$ case once we produce a result emulating Proposition A.5 in 3D. By Proposition A.4 we get the existence of a sequence $\left\{\zeta_{n}\right\}_{n}$ which satisfies (up to the transformation $x \rightarrow$ $\left.x_{0}+r_{0}\left(x-x_{0}\right)\right)$ the properties listed in the proposition below.

Proposition A.6. Let $x_{0} \in \mathbb{R}^{3}, r_{0}>0$ and $M: B_{0}:=B\left(x_{0}, r_{0}\right) \rightarrow \mathcal{S}_{3}(\mathbb{R})$ be continuous. Denote by $Q_{0}$ the constant orthogonal matrix such that $Q_{0} M\left(x_{0}\right) Q_{0}^{T}$ is diagonal. Let $\left\{\zeta_{n}\right\}_{n} \in C_{c}^{\infty}\left(B_{0} ; \mathbb{R}^{3}\right)$ be such that

$$
\operatorname{spt}\left(\zeta_{n}\right) \subset B\left(x_{0}, r_{n}\right) \text { for a decreasing sequence }\left\{r_{n}\right\}_{n} \downarrow 0
$$

and

$$
\frac{\left|\left(Q_{0} \nabla \times \zeta_{n}\right)_{2}\right|+\left|\left(Q_{0} \nabla \times \zeta_{n}\right)_{3}\right|}{\left\|\nabla \times \zeta_{n}\right\|_{H^{1}\left(B_{0}\right)}} \rightarrow 0 \text { as } n \rightarrow \infty
$$

If

$$
\int_{B_{0}} M(x) \nabla \times \zeta_{n}(x) \cdot \nabla \times \zeta_{n}(x) \mathrm{d} x \geq 0 \text { for all } n \geq 1
$$

then the first eigenvalue of $M\left(x_{0}\right)$ is nonnegative.

Proof. The characteristic polynomial of $M$ is

$$
g(\lambda):=-\lambda^{3}+(\operatorname{tr} M) \lambda^{2}+\frac{1}{2}\left[(\operatorname{tr} M)^{2}-\operatorname{tr}\left(M^{2}\right)\right] \lambda+\operatorname{det} M
$$

The discriminant of the equation $-g_{0}(\lambda)=0$ is

$$
D:=18 b c d-4 b^{3} d+b^{2} c^{2}-4 c^{3}-27 d^{2},
$$

where $b:=-\operatorname{tr} M, c:=-\left[(\operatorname{tr} M)^{2}-\operatorname{tr}\left(M^{2}\right)\right] / 2, d:=-\operatorname{det} M$. It is well-known that $D \geq 0$ is equivalent to all roots being real. We may also assume that all the entries of $M$ are nonzero. This is because there exists $\delta_{0}>0$ such that for all $0<\delta \leq \delta_{0}$ we have $M_{i j}+\delta \neq 0$ for all $1 \leq i, j \leq 3$. Let $\Phi_{\delta}(x):=\delta\left(x_{1}+x_{2}+x_{3}\right)^{2} / 2$; then $M+\nabla^{2} \Phi_{\delta}$ satisfies (A.21) as well, and its eigenvalues converge to the eigenvalues of $M$. Thus, it is enough to prove that the first eigenvalue of this perturbed matrix (at $x_{0}$ ) is nonnegative for all $\delta_{0} \geq \delta>0$. Since for all $\delta$ in this range we have that all the entries of $M\left(x_{0}\right)+\nabla^{2} \Phi_{\delta}\left(x_{0}\right)$ are nonzero, we may now work under that assumption. However, it turns out it is more convenient to only assume, which we do, that the off-diagonal entries of $M\left(x_{0}\right)$ are nonzero. 
1. Assume $D\left(x_{0}\right)>0$, i.e. the three real roots are distinct (another well-known fact), and the top two rows of the matrix $M\left(x_{0}\right)-\lambda I_{3}$ are linearly independent for any eigenvalue $\lambda$. The latter is equivalent to

$$
\text { either } \frac{m_{11}-\lambda}{m_{12}} \neq \frac{m_{13}}{m_{23}} \text { or } \frac{m_{22}-\lambda}{m_{12}} \neq \frac{m_{23}}{m_{13}} \text { for any eigenvalue } \lambda \text {. }
$$

For each eigenvalue $\lambda$ we look for an eigenvector $\mathbf{q} \in \mathbb{R}^{3}$ by singling out a nontrivial solution of the system

$$
\left(m_{11}-\lambda\right) q_{1}+m_{12} q_{2}+m_{13} q_{3}=0, m_{12} q_{1}+\left(m_{22}-\lambda\right) q_{2}+m_{23} q_{3}=0 .
$$

If

$$
\frac{m_{11}-\lambda}{m_{12}} \neq \frac{m_{13}}{m_{23}}
$$

we do this by setting $q_{2}=-1$ and then solving to find

$$
q_{1}=\frac{m_{13} \lambda+m_{12} m_{23}-m_{13} m_{22}}{m_{11} m_{23}-m_{12} m_{13}-m_{23} \lambda}, q_{3}=\frac{m_{22}-\lambda-m_{12} q_{1}}{m_{23}},
$$

which are valid (denominators are nonzero). Instead, if

$$
\frac{m_{11}-\lambda}{m_{12}}=\frac{m_{13}}{m_{23}}
$$

according to (A.23), we do this by setting $q_{1}=-1$ and then solving to find

$$
q_{2}=\frac{m_{23} \lambda+m_{12} m_{13}-m_{11} m_{23}}{m_{22} m_{13}-m_{12} m_{23}-m_{13} \lambda}, q_{3}=\frac{m_{11}-\lambda-m_{12} q_{2}}{m_{13}} .
$$

We normalize these values into the unit vector $\mathbf{q}_{\lambda}$. Since the eigenvalues are distinct, the three vectors $\mathbf{q}_{\lambda}$ do form an orthonormal set of vectors. Again, by the continuity of the eigenvalues with respect to the coefficients, we infer that we still have distinct real roots and the linear independence of the top two rows of $M(x)-\lambda I_{3}$ still holds for all eigenvalues $\lambda$ (and, consequently, the above choices of eigenvectors still works) if we replace $M\left(x_{0}\right)$ by $M(x)$ for all $x$ in a sufficiently small ball around $x_{0}$, say $B\left(x_{0}, 2 r\right)$. We define the matrix $Q$ whose columns are the vectors $\mathbf{q}_{\lambda}$ computed at $x \in \overline{B\left(x_{0}, r\right)}$; due to the above considerations, $Q$ is a continuous function on $\overline{B\left(x_{0}, r\right)}$. So, one can still perform a decomposition $M=Q^{T} \Lambda Q$ which is continuous in $\overline{B\left(x_{0}, r\right)}$. This means that if $\lambda_{1}\left(x_{0}\right)<0$, we can use (A.21) and imitate the 2D case to conclude that there is $r \geq s_{0}>0$ such that $B_{0}:=B\left(x_{0}, s_{0}\right) \subset \Omega$ and

$$
\int_{B_{0}}\left|\mathbf{v}_{n}\right|^{2} \mathrm{~d} x \leq \lambda_{0} \int_{B_{0}}\left[\left|\left(Q_{0} \mathbf{v}_{n}\right)_{2}\right|^{2}+\left|\left(Q_{0} \mathbf{v}_{n}\right)_{3}\right|^{2}\right] \mathrm{d} x
$$

for all $n$ sufficiently large such that $\zeta_{n}$ is supported in $B_{0}$, where $\lambda_{0}$ is a positive constant and $\mathbf{v}_{n}:=\nabla \times \zeta_{n}$. This contradicts (A.20).

2. Now we only assume $D\left(x_{0}\right)>0$. If the first two rows of $M\left(x_{0}\right)$ are linearly dependent for some eigenvalue $\lambda$, then

$$
\lambda=m_{11}-\frac{m_{12}}{m_{23}} m_{13}=m_{22}-\frac{m_{12}}{m_{13}} m_{23},
$$


which implies $m_{22}-m_{11}=m_{12}\left(m_{23}^{2}-m_{13}^{2}\right) /\left(m_{13} m_{23}\right)$. However, note that by adding $\delta>0$ to $m_{11}$ this equality is violated, and for all $\delta>0$ sufficiently small $D\left(x_{0}\right)$ stays positive if we replace $m_{11}$ by $m_{11}+\delta$. Since for $\bar{\Phi}_{\delta}(x):=$ $\delta x_{1}^{2} / 2, M+\nabla^{2} \bar{\Phi}_{\delta}$ satisfies (A.21) if $M$ does, it is enough to prove that the eigenvalues of $M\left(x_{0}\right)+\nabla^{2} \bar{\Phi}_{\delta}\left(x_{0}\right)$ are nonnegative for all sufficiently small $\delta>0$ in order to conclude (via the continuity of eigenvalues with respect to matrix entries) that $\lambda_{1}\left(x_{0}\right) \geq 0$. But $M\left(x_{0}\right)+\nabla^{2} \bar{\Phi}_{\delta}\left(x_{0}\right)$ satisfies the restrictions imposed in part 1 of this proof, so we are done (if $D\left(x_{0}\right)>0$ ).

3. All that is left is the case $D\left(x_{0}\right)=0$. Since $M$ cannot be a scalar multiple of the identity matrix (which would violate the assumption that all off-diagonal entries are nonzero), one of the quantities $\left(m_{11}-m_{22}\right)^{2}+4 m_{12}^{2}$, $\left(m_{22}-m_{33}\right)^{2}+4 m_{23}^{2}$ and $\left(m_{11}-m_{33}\right)^{2}+4 m_{13}^{2}$ is nonzero. Without loss of generality, assume that $\left(m_{11}-\right.$ $\left.m_{22}\right)^{2}+4 m_{12}^{2}>0$. Let $\tilde{\Phi}_{\delta}(x):=\delta x_{3}^{2} / 2$ so that $M\left(x_{0}\right)+\nabla^{2} \tilde{\Phi}_{\delta}\left(x_{0}\right)$ is also a symmetric real matrix, so the discriminant of its characteristic polynomial is also nonnegative; some elementary calculations lead to (with the notation from (A.22))

$$
\begin{aligned}
D^{\delta}:= & 18(b-\delta)(c-\alpha \delta)(d-\beta \delta)-4(b-\delta)^{3}(d-\beta \delta) \\
& +(b-\delta)^{2}(c-\alpha \delta)^{2}-4(c-\alpha \delta)^{3}-27(d-\beta \delta)^{2}=: h(\delta),
\end{aligned}
$$

for $\alpha:=m_{11}+m_{22}, \beta:=m_{11} m_{22}-m_{12}^{2}$. We see that $h$ is a polynomial in $\delta$ of degree four (since its dominant coefficient is $\left.\left(m_{11}-m_{22}\right)^{2}+4 m_{12}^{2}>0\right)$ such that $h(\delta) \geq 0$ for all $\delta \in \mathbb{R}$ and $h(0)=D\left(x_{0}\right)=0$; we conclude that $h$ has at most three more real roots, so there exists $\delta_{0}>0$ such that $h(\delta)>0$ for all $\delta_{0} \geq \delta>0$, which is equivalent to the roots of the characteristic polynomial of $M\left(x_{0}\right)+\nabla^{2} \tilde{\Phi}_{\delta}\left(x_{0}\right)$ being real and distinct. Since $M+\nabla^{2} \tilde{\Phi}_{\delta}$ satisfies (A.21) as well, we are now in the setting of part 2, so all the first eigenvalue of $M\left(x_{0}\right)+\nabla^{2} \tilde{\Phi}_{\delta}\left(x_{0}\right)$ is nonnegative. We conclude the proof by letting $\delta \rightarrow 0^{+}$and using, once again, the continuity of the eigenvalues with respect to the matrix entries.

Acknowledgements. Adrian Tudorascu was partially supported by the National Science Foundation under Grant DMS1600272. We would like to thank the anonymous referee for suggesting the constructive method for proving Theorem 2.5.

\section{REFERENCES}

[1] L. Ambrosio, M. Colombo, G. De Philippis and A. Figalli, Existence of Eulerian solutions to the semigeostrophic equations in physical space: the 2-dimensional periodic case. Commun. Part. Differ. Equ. 37 (2012) 2209-2227.

[2] L. Ambrosio, M. Colombo, G. De Philippis and A. Figalli, A global existence result for the semigeostrophic equations in three dimensional convex domains. Discrete Contin. Dyn. Syst. 34 (2014) 1251-1268.

[3] J.-D. Benamou and Y. Brenier, Weak existence for the Semi-Geostrophic equations formulated as a coupled MongeAmpere/transport problem. SIAM J. Appl. Math. 58 (1998) 1450-1461.

[4] Y. Brenier, Polar factorization and monotone rearrangement of vector-valued functions. Comm. Pure Appl. Math. 44 (1991) 375-417.

[5] Y. Brenier and M. Cullen, Rigorous derivation of the $X-Z$ Semigeostrophic equations. Commun. Math. Sci. 7 (2009) $779-784$.

[6] P. Cardaliaguet, Notes on Mean-Field Games, lectures by P.L. Lions, Collège de France (2010).

[7] J. Cheng, M. Cullen and M. Feldman, Semi-Geostrophic system with variable Coriolis parameter. Arch. Ration. Mech. Anal. 227 (2018) 215-272.

[8] G. Crippa, The flow associated to weakly differentiable vector fields. Ph.D. thesis, Scuola Normale Superiore di Pisa (2008).

[9] M.J.P. Cullen, A mathematical theory of large-scale atmosphere/ocean flow. Imperial College Press (2006).

[10] M.J.P. Cullen, T. Kuna, B. Pelloni and M. Wilkinson, The Stability Principle and global weak solutions of the free surface semi-geostrophic equations in geostrophic coordinates. Proc R Soc A 475 (2019) 20180787.

[11] M.J.P. Cullen and M. Feldman, Lagrangian solutions of Semi-Geostrophic equations in physical space. SIAM J. Math. Anal. 37 (2006) 1371-1395.

[12] M.J.P. Cullen and W. Gangbo, A variational approach for the 2-dimensional semi-geostrophic shallow water equations. Arch. Rat. Mech. Anal. 156 (2001) 241-273.

[13] G. De Philippis and A. Figalli, $W^{2,1}$ regularity for solutions of the Monge-Ampère equation. Invent. Math. 192 (2013) 55-69.

[14] J.C. O Faria, M.C. Lopes Filho and H.J. Nussenzveig Lopes, Weak stability of Lagrangian solutions to the Semi-Geostrophic equations. Nonlinearity 22 (2009) 2521-2539.

[15] M. Feldman and A. Tudorascu, On Lagrangian solutions for the Semi-Geostrophic system with singular initial data. SIAM J. Math. Anal. 45 (2013) 1616-1640. 
[16] M. Feldman and A. Tudorascu, On the Semi-Geostrophic system in physical space with general initial data. Arch. Rat. Mech. Anal. 218 (2015) 527-551.

[17] M. Feldman and A. Tudorascu, The Semi-Geostrophic system: weak-strong uniqueness under uniform convexity. Calc. Var. Partial Differ. Equ. 56 (2017) 158.

[18] B. Hoskins, The geostrophic momentum approximation and the semi-geostrophic equations. J. Atmos. Sci. 32 (1975) $233-242$.

[19] T. Kato, Perturbation Theory for Linear Differential Operators. Springer (1995).

[20] G. Loeper, A fully nonlinear version of the incompressible Euler equations: the Semigeostrophic system. SIAM J. Math. Anal. 38 (2006) 795-823. 